\title{
Studying neuroanatomy using MRI
}

Jason P. Lerch ${ }^{1,2}$, André J.W. van der Kouwe ${ }^{3,4}$, Armin Raznahan ${ }^{5}$, Tomas Paus ${ }^{6,7,8}$, Heidi Johansen-Berg ${ }^{9}$, Karla L. Miller ${ }^{9}$, Stephen M. Smith ${ }^{9}$, Bruce Fischl ${ }^{3,4,10}$, Stamatios N. Sotiropoulos ${ }^{9,11}$.

${ }^{1}$ Program in Neuroscience and Mental Health, The Hospital for Sick Children, Toronto, ON, Canada

${ }^{2}$ Department of Medical Biophysics, University of Toronto, Toronto, ON, Canada

${ }^{3}$ Athinoula A. Martinos Center for Biomedical Research, Department of Radiology, Massachusetts General Hospital and Harvard Medical School, Charlestown, MA, USA

${ }^{4}$ Department of Radiology, Massachusetts General Hospital and Harvard Medical School, Boston, MA, USA

${ }^{5}$ Developmental Neurogenomics Unit, Child Psychiatry Branch, National Institute of Mental Health, Bethesda, MD, USA

${ }^{6}$ Rotman Research Institute, Baycrest, Toronto, ON, Canada

${ }^{7}$ Departments of Psychology and Psychiatry, University of Toronto, Toronto, ON, Canada

${ }^{8}$ Center for the Developing Brain, Child Mind Institute, New York, NY, USA

${ }^{9}$ Oxford Centre for Functional MRI of the Brain (FMRIB), University of Oxford, Oxford, UK

${ }^{10}$ Computer Science and Artificial Intelligence Laboratory, Massachusetts Institute of Technology, Cambridge, MA, USA

${ }^{11}$ Sir Peter Mansfield Imaging Centre, School of Medicine, University of Nottingham, Nottingham, UK

\section{Abstract}

The study of neuroanatomy using imaging enables key insights into how our brains function, are shaped by genes and environment, and change with development, aging, and disease.

Developments in MRI acquisition, image processing, and data modelling have been key to these advances. However, MRI provides an indirect measurement of the biological signals we aim to investigate. Thus, artifacts and key questions of correct interpretation can confound the readouts provided by anatomical MRI. In this review we provide an overview of the methods for measuring macro- and mesoscopic structure and inferring microstructural properties; we also describe key artefacts and confounds that can lead to incorrect conclusions. Ultimately, we believe that, though methods need to improve and caution is required in its interpretation, structural MRI continues to have great promise in furthering our understanding of how the brain works. 


\section{Introduction}

The study of neuroanatomy can be traced back to ancient Egypt, and brought into the modern era by the seminal tracings of the brain and nerves by Thomas Willis. The brain was long viewed as the seat of consciousness and thought. A direct linkage between cognition and individual brain areas arose in the 19th century, as epitomized by the work of Broca, Wernicke, and Lichtheim ${ }^{1}$. Detailed studies of the composition of cell types across regions of the brain by Ramon y Cajal, von Economo, Brodmann, and others then laid the foundation of our current understanding of the anatomy of the central nervous system (reviewed in ${ }^{1}$ ). With the increasing certainty of the localization of function to specific brain regions came questions about the evolution of these brain regions. This in turn led to questions about the possible relationship between their size or shape and inter-individual variations in cognitive abilities or progression of a disease.

The advent of Magnetic Resonance Imaging (MRI) in the late 70 s and early 80 s revolutionized the study of neuroanatomy, as for the first time it could be investigated in-vivo with sufficient contrast to differentiate brain compartments. The 90s introduced computational approaches to the MRI-based studies of neuroanatomy. At the same time, novel quantitative, or semi-quantitative, MRI techniques emerged, allowing one to estimate microstructure within a voxel. The advent of automated processing pipelines further enabled large scale analyses of population imaging data in ways that were previously time-prohibitive.

The ability to obtain high-quality, detailed information from in-vivo imaging has clearly revolutionized our understanding of neuroanatomy and structure-function relations, and shed insights into multiple disease processes. Yet there are myriads of issues surrounding acquisition, analysis, study design and interpretation that need to be considered. This review provides readers with an overview of these issues in order to equip them to select the most appropriate toolkit for their needs.

\section{Overview of the methods}

Key to understanding MRI based measures of neuroanatomy is that, with MRI, we are not directly measuring the cellular compartments we would like to make inferences on. Limited resolution, combined with indirect measurements, caution against simplistic extrapolation from MRI findings to neurobiological conclusions. In contrast to the common metaphor of "taking a picture of the brain", MRI instead measures radio-frequency signals emitted from hydrogen atoms after the application of electromagnetic (radio-frequency) waves, with the signal being localized using spatially varying magnetic gradients. Contrast from each voxel (a threedimensional pixel) depends on the density of protons within the voxel and properties of the local tissue microenvironment that are either directly related to the magnetic properties of hydrogen or which can be detected through manipulation of magnetic fields. The contrast produced is dependent on the precise timing of the magnetic field manipulations (pulse sequences), as shown in figure 1. 
To provide more detail, tissue contrast is dependent on $T_{1}$ and $T_{2}$ relaxation, two independent processes that describe how spins (i.e. magnetic properties) behave after the application of radio-frequency pulses. $T_{1}$ represents the time constant for a system of hydrogen protons to return to equilibrium after radio-frequency radiation ${ }^{2} . T_{1}$ values are determined by macro-molecule concentration, water binding, and water content ${ }^{3}$, with myelin shortening $T_{1}$ and oedema lengthening $T_{1}$. T2, or transverse, relaxation describes the process by which spins are taken out of alignment with each other due to variations in the local tissue environment at the micro- or nanoscopic scale ${ }^{4}$. T2 effects are observed as signal decay, with grey matter having a longer $\mathrm{T}_{2}$ relaxation time than white matter. Structural MRI studies almost universally use "weighted" imaging, where the signal intensity is related to T1 and T2, rather than quantifying these or other properties (because these are generally more efficient, giving greater signal to noise for a given acquisition duration).

Once images have been acquired, measuring brain structure involves two complementary approaches: (1) the macro- or mesoscopic, considering sizes and shapes across multiple voxels, and (2) the microscopic, obtaining information from within voxels.

\section{Macro- and meso-scopic neuroanatomy}

There are five domains to studying size and shape at the macro- or mesoscopic scale:

(1) Manual volumetry, involves trained anatomists manually segmenting regions of interest from brain scans. In many ways still the gold standard, it is hard to scale to multiple scans (especially for modern studies running into the thousands of participants) ${ }^{5,6}$ and multiple brain regions. Thus, the primary use of manual volumetry is in smaller studies with a single, focused hypothesis, as well as for the creation of datasets to be incorporated into automatic segmentation algorithms.

(2) Automatic segmentation algorithms aim to replace manual volumetry for most applications. In some cases manually segmented datasets are used to parcellate new data based on some combination of linear and non-linear image registration, tissue classification, and related image features, while for other algorithms no manual training is necessary. The time of trained anatomists is traded for computer time, with computationally intensive multi-atlas approaches and/or additional surface based constraints adding extra accuracy ${ }^{7}$. Agreement between manual volumetry and automatic segmentation indicates ever improving correspondence between the two.

(3) Two classes of morphometry algorithms are commonly used to analyze neuroanatomy without the prior constraint of defined regions of interest: voxel-based morphometry (VBM) and deformation-based morphometry (DBM). In VBM, illustrated in figure 2, the brain is classified into white matter, grey matter, and cerebrospinal fluid, a single tissue class selected, then blurred with a Gaussian kernel to give an estimate of the local amount of that tissue type at every voxel, then compared across subjects after linear or coarse non-linear alignment $^{8}$. In DBM, brains are non-linearly deformed towards a common space or each other, and deformation fields are either directly analyzed ${ }^{9}$ or reduced to a scalar measure of volume change, the Jacobian determinant, before comparisons across scans ${ }^{10}$. These 
two methods are joined in optimized VBM, wherein the tissue density measure is modulated by the Jacobian determinant ${ }^{11}$.

(4) The complex folding pattern of the cerebral cortex challenges computational neuroanatomy, though that significantly improved with the development of surface-based algorithms, as shown in figure 3 . Here the inside and outside surfaces of the cortex are segmented using deformable models and measures such as cortical thickness and surface area are extracted ${ }^{12-14}$. The diverse anatomical metrics that can be extracted from threedimensional models of the cortical surface capture different developmental processes, and show dissociable correlations with demographic ${ }^{15,16}$, genetic $^{17,18}$, environmental ${ }^{19}$, and clinical $^{20,21}$ variables - highlighting the value in moving classical volumetric approaches to anatomical analysis. Surface based methods also provide an improved coordinate system for the cerebral cortex, allowing for smoothing of signal on the cortical sheet and surface based alignment to bring individuals into closer correspondence for statistical comparisons $^{13,22}$.

(5) Estimation of white-matter tract locations and sizes using diffusion tractography. Here measures of water diffusion, described in greater detail below, are used to trace the pathways of the brain, followed by inferences on properties of each tract. The benefits and caveats of tracing the macroscopic connections of the brain were described in greater detail in a recent Nature Neuroscience review ${ }^{23}$, to which we refer the reader for a more indepth description.

Since MRI is not a direct picture of the underlying anatomy the outcomes of derived measures, such as cortical thickness or VBM, depend on sequence and hardware choices ${ }^{24,25}$. Measures of cortical thickness at $7 \mathrm{~T}$ are, for example, lower than when measured at $3 \mathrm{~T}^{26}$. Varying tissue properties across the cerebral cortex present different degrees of challenge in separating grey from white matter; the motor cortex is heavily myelinated in its lower layers, and thus MR based thickness estimates (especially in early studies) tend to underestimate its thickness compared with histological measurements ${ }^{27}$. Constant improvements in hardware along with MR-sequence development are bringing imaging measures ever closer to post-mortem ground truth, but the nature of the MR signal needs always to be kept in mind when offering interpretations of imaging data; what we measure is not always identical to what the anatomists of old (and today) would have estimated. This becomes an even greater issue when potential artefacts segregate with the study population, as discussed later in this review.

\section{Microstructure - MRI at the microscopic level}

The study of microstructure - which in MRI refers to the distribution of the contents of a voxel has been primarily the domain of diffusion MRI. Here the thermally-driven, random motion of water molecules is a probe of the local microenvironment, and the restriction of that motion is used to infer the organization of the tissue inside the imaging voxel.

Initial studies modelled the scatter pattern of diffusing water molecules with an ellipsoidal shape, represented by a tensor ${ }^{28}$. Various metrics can be derived from the diffusion tensor, such as the fractional anisotropy (FA, representing how elongated the shape is), as well as the mean, axial, and radial diffusivity (what is the radius of the ellipse along different axes) ${ }^{29}$. 
Variations in these metrics reflect variations in the profile of water diffusion and have been therefore associated with alterations in the underlying tissue microstructural boundaries. They can be compared across individuals at every voxel after alignment (e.g. ${ }^{30}$ ), or averaged across tracts after tractography-based tract segmentation (e.g. ${ }^{31}$ ). To overcome registration issues, skeleton-based approaches were introduced, whereby values are projected to an alignment invariant mean-FA skeleton representing the center of major tracts, which also eliminates partial volume effects at the edge of tracts ${ }^{32,33}$.

Tensor-based modelling of diffusion MRI data, while sensitive to alterations in microstructure, is not specific to a given types of variation in tissue properties ${ }^{29}$. Furthermore, in regions with complex fibre patterns or significant restriction (e.g. entrapment of water in a particular cellular compartment), the tensor model does not capture the underlying structure and only provides an average unimodal approximation.

Acquiring multiple "shells" - diffusion MRI acquisition with different sensitivity to diffusion process (typically quantified by the experimental parameter $b$ ) - opens up further potential for analyses. Multi-shell measurements enable deviations from the tensor model (and therefore complex microstructure) using higher-order approximations, such as the diffusion kurtosis model $^{34}$. Even kurtosis estimates of diffusion measures still lack direct biological interpretation, similar to parameters in the tensor model; however, model-based mappings ${ }^{35}$ offer potential work-arounds (see also Box 1). As both modelling and acquisition methods evolve, estimates with greater specificity can be obtained. Possible inferences range for instance from mapping crossing-fibres in white matter to estimating neurite densities in grey matter. These methods are summarized in Box 1.

While diffusion based approaches have become the dominant method for inferring microstructure, other quantitative imaging techniques still provide unique information. Magnetization transfer (MT) imaging reflects the interaction between free-water protons and protons bound to large macromolecules ${ }^{36}$, such as proteins. MT is often employed in studies of the structural properties of white matter, where the macromolecules of myelin are the dominant source of the signal ${ }^{37}$. MT is also used to probe damaged tissue and inflammation due to its sensitivity to protein content ${ }^{38}$. The fastest way to acquire magnetization-transfer data is to use two acquisitions, with and without a MT saturation pulse, and thereafter calculate the magnetization transfer ratio as the percent signal change between the two acquisitions ${ }^{39}$. A variant of MT is Chemical Exchange Saturation Transfer (CEST) ${ }^{40}$, with application for detecting $\mathrm{pH}$ changes in stroke ${ }^{41}$ and tumour delineation ${ }^{42}$.

Instead of using "weighted" images for subsequent image processing, one can also quantify $T_{1}$ and $T_{2}$ directly. The aim is to allow greater inference about local tissue composition, for example the very short $\mathrm{T}_{2}$ associated with myelin compared to other water within a voxel ${ }^{43}$. The main downside of quantitative $T_{1}$ and $T_{2}$ mapping is the significantly greater amount of time required for equivalent SNR and resolution, as the gold-standard methods require acquiring data at multiple echos or inversion times ${ }^{43,44}$. This is compounded by the general need to estimate multiple $T_{2}$ values within each voxel if one wants to map specific compartments like myelin. Faster approximations exist ${ }^{45-47}$, yet these methods have significant variation in reported metrics such that calibration to the gold standard is still required ${ }^{48}$.

The sequences described above all rely on the magnitude information and discard phase information. Phase information can, however, be used to identify susceptibility changes 
between tissues. For example, phase images can be used to quantify the mean magnetic susceptibility of the tissue in a voxel, which is arguably a more direct measure of the tissue magnetism than many of the relaxation time-based methods described above. This method, known as quantitative susceptibility mapping (QSM) has been demonstrated to change with tissue iron, myelin and calcium.

\section{Applications of structural MRI}

The spatial organization of the brain, and its microstructural properties, are the physical manifestation of the information encoded in the genome, developmental history, and experienced environment, and thus touches on just about every facet of neuroscience. As described above, MRI enables the derivation of multiple (semi-)quantitative features of brain structure in a reliable manner (test-retest correlations $>0.75)$, over short time ( $\sim 1$ hour) and on a large scale (>1,000 participants). It is clearly impossible, within this review, to provide an overview of all aspects of neuroscience touched on by MRI studies of brain structure. Instead we provide two illustrative examples. The first relates to combining genetics and epidemiology with brain imaging to study populations (see Box 2). Secondly, inter-individual differences in adult cognition, behavior and psychiatric disease can often be linked to earlier developmental variations that emerge across infancy, childhood and adolescence. Structural neuroimaging plays a special role in unraveling these developmental associations since changes in brain structure work across both short and long time-scales (see Box 3).

Finally, brain structure need not be only analyzed as a series of independent segmented regions or even voxels. Instead neuroanatomy can also be studied in terms of networks of covariance. Studying brain networks using brain imaging first came out of activational and resting state fMRI or PET studies. Similar networks emerged when correlating seed regions with the rest of the brain using either $\mathrm{VBM}^{49}$ or cortical thickness ${ }^{50}$ across a population of subjects. Variations in such structural covariance networks have been linked to both normal development, aging, as well as multiple disease processes ${ }^{51,52}$. The link to other measures of networks,

whether from functional imaging or tract tracing is, however, ambiguous ${ }^{53}$, and clearly more work is needed to understand the origins and significance of structural covariance networks.

\section{Forward problem, inverse problem: what are the signal sources, and what kind of inferences can we make?}

Once imaging results have identified a finding of interest, establishing their molecular and cellular bases is often elusive. The study of brain plasticity with MRI provides an illustrative example. The early identification of altered "concentration" of hippocampal grey-matter (as measured by VBM) in London taxi drivers suggested that the processes of learning and memory, so long studied in animal models, might be detectable by MRI in humans ${ }^{54}$. Additional evidence came from studies showing that teaching adults how to juggle altered grey-matter 
concentrations in the visual and parietal cortex ${ }^{55}$, a change that occurred in as quickly as seven days $^{56}$, and was accompanied by changes in water diffusion in underlying white matter ${ }^{57}$. Microstructural changes have even been detected using diffusion MRI within two hours of playing a video game ${ }^{58}$. Further work by multiple groups has shown that MRI can detect brain plasticity in motor coordination tasks ${ }^{59}$ and musical training ${ }^{60}$ amongst others. Intriguing as they are, these studies are not without some refutation and debate. For example, one danger of misinterpretation can be seen in Bengtsson et al. ${ }^{61}$, where reported areas of change after piano practice are mostly on the very edge of the white matter tracts, and therefore likely represent changes in tract thickness rather than microstructural properties of the tracts. Juggling, the most studied training task for inducing structural brain plasticity, often shows changes in the visual and parietal cortices, but the precise localization of those effects is poorly reproducible ${ }^{62}$. Detecting brain plasticity in a study of adults trained on a joystick task was found to be extremely dependent on methodological choices made during image processing and appeared to be artifactual $^{63}$.

Studies of human brain plasticity were then back-translated into animal models, in particular mice and rats. The advantage of rodent models is, of course, that invasive histology/immunohistochemistry experiments can be carried out after MRI assessments. Initially, it was established that learning causes changes in regional brain volumes ${ }^{64}$ and diffusion properties ${ }^{65}$. Such alterations could be detected within a day and yet seemed to last for weeks or months ${ }^{66}$. At the level of correlations, diffusion properties changes in white matter were correlated with myelin markers ${ }^{67}$; diffusion in grey matter correlated with astrocytic and synaptic markers ${ }^{65}$. Volumetric changes similarly correlated with synaptic markers, in particular GAP43, an axonal growth cone indicator ${ }^{64,68}$, dendritic spine counts ${ }^{69}$, and glutamate concentration ${ }^{70}$. Establishing more causal connections between cellular and mesoscopic changes is just beginning; irradiation, for example, has been shown to stop both neurogenesis and exercise-related increases hippocampal volume ${ }^{71}$. Genetic mouse models in particular promise to play an important role in linking molecular mechanisms to MRI outcomes.

Even with using animal models to provide direct mechanistic explanations of MRI signal, interpretation will remain contextual. An increase in grey matter in the hippocampus might be conclusively linked to synaptic changes in the future, but only within the context of healthy individuals engaging in a learning task. In pathological states, the same change in hippocampal volume could be caused by entirely unrelated cellular mechanisms. Advanced MR sequences, in particular microstructure modelling approaches, offer the promise of greater specificity than volumetric measures. Working with a rodent model, Jespersen ${ }^{72}$ compared ex-vivo multi-shell diffusion data with a combination of myelin and cell body staining. They identified a high degree of correlation between neurite density (estimated from advanced diffusion MRI models) and myelin maps; moreover, the biophysical models based on assumptions regarding cellular structure clearly outperformed the simpler tensor model ${ }^{72}$. Similarly, related work identified a strong relationship between myelin density in the corpus callosum as measured using a modified variant of the NODDI model (see Box 1) and electron microscopy ${ }^{73}$. Combining quantitative magnetisation transfer with multi-shell diffusion imaging, Stikov and colleagues showed that the g-ratio, the ratio of the inner to outer diameter of the myelin sheath, could be estimated accurately as compared against electron microscopy ${ }^{74}$. These examples illustrate that the field is moving ever closer to accurate estimates of direct measures of neural morphometry, 
though clearly much more work is needed to establish both levels of accuracy and robustness of existing measures and extend into new biology-based indices. We need to keep in mind that an indirect mapping/inference is necessary to go from what we measure to tissue biophysical properties in order to avoid over-interpretation of results ${ }^{75}$.

\section{An expensive motion detector? MRI artifacts and caveats}

MRI is not a simple "picture of the brain", which has advantages viz. the multiple contrasts that can be obtained by different pulse sequences, but also leads to a series of possible artifacts that can confound easy interpretation of results and, at their worst, even lead to entirely incorrect conclusions. The ubiquitous and varied nature of artifacts in MR imaging stems from the fundamental physical difficulty of manipulating the magnetic field (B0) to be either uniform in space or form linear intensity ramps, and manipulating radio-frequency (RF) fields (B1) to be uniform in space. Furthermore, these fields are required to be rapidly, accurately and reproducibly modulated in time to encode the image. These requirements are compounded by the fact that data encoding occurs in the spatial frequency domain, resulting in MR artifacts having quite different characteristics than one might encounter in a camera or other imaging system. Additionally, even subtle inter-subject differences, such as hydration levels ${ }^{76}$ or time of day ${ }^{77}$ influence outcomes. Lastly, the image processing, and particularly spatial normalization, used to derive the metrics that go into the ultimate statistical analyses also come with potential confounds. Thus, when performing a morphometry study it is critical to identify the potential sources of artifacts, and explicitly investigate whether they may be the underlying source of a perceived biological effect when confounds segregate with predictors (i.e. patients moving more than controls), so as to not end up using MRI as an overpriced motion detector.

\section{Subject Motion}

The most common potential confound in morphometry studies is subject motion. While the fact that subject motion can contaminate or even induce MRI findings has been known for decades, the subtle and pernicious nature of motion confounds has only recently been quantified. The heightened concern regarding motion related biases in neuroimaging first emerged in the context of functional neuroimaging data ${ }^{78}$, and then rapidly extended into the structural neuroimaging community ${ }^{79,80}$. Accounting for subject motion is fundamentally difficult because (i) acquisition is performed in the spatial frequency domain, therefore the effects of motion on the reconstructed image will vary depending on the timing, direction and amount of motion, and (ii) in the vast majority of group studies the amount of motion will be correlated with the effect we are trying to study (e.g. elderly subjects move more than middle aged, subjects with more advanced diseases move more than those earlier in the course, subjects may move differently with medication). Further, it has been shown ${ }^{79}$ that motion changes the information content of the images in the direction we would typically expect in an atrophy study - more motion induces an apparent reduction in gray matter that is difficult to distinguish from true atrophy. For diffusion MRI-derived microstructure estimates, motion can also induce spurious differences 
between groups, even in cases of comparing groups with control subjects only, when no differences are expected ${ }^{81}$. These phenomena pose special challenges for the collection, analysis and interpretation of developmental neuroimaging data given that (i) motion is nonrandomly distributed with respect to age (children>adults), sex (male>female), and clinical status $^{82}$, and (ii) motion-induced biases are prominent in brain regions (e.g. prefrontal cortices) that are notable for displaying anatomical differences as a function of age, sex and disease status $^{80}$. Indeed, the adoption of approaches to excluding scans based on motion artifact impacts conclusions regarding trajectories of neuroanatomical change in brain development ${ }^{15}$.

Approaches for addressing motion-related confound in neuroimaging can be divided into post-hoc strategies that attempt to minimize the impact of motion on already gathered images, and prospective strategies that seek to reduce motion contamination during image acquisition. The most basic post-hoc strategy is to estimate the amount of motion in each scan and then exclude any scans which exceeding a set motion cut-off and/or match motion magnitude in individuals across groups. This of course can be inefficient as it requires discarding one's best data at times and can rule out certain designs. Estimates of in-scanner motion could potentially also be used as regressors in statistical analysis in an attempt to "control" for motion effects ${ }^{81}$.

For diffusion MRI, where multiple measurements are obtained for each voxel, motion can blur the estimated maps, due to misalignment of the measured volumes. Furthermore, subject motion can be difficult to estimate, as it interacts with hardware-related artifacts (eddy currents) and field-inhomogeneity distortions (see next section). However, recent frameworks offer improved and robust performance in detecting and correcting these artifacts (e.g. ${ }^{83}$ ). After having estimated motion and artifacts, one can detect outlier data ${ }^{84}$ and either downweight them or replace them with average predictions obtained from non-outlier measurements to minimise bias and effects on subsequent analysis ${ }^{85}$.

In contrast to these post-hoc approaches, prospective strategies seek to detect and account for subject motion during the acquisition itself. Approaches to motion detection/correction take two basic forms - (1) the detection is provided by an external tracking device, or (2) the MRI signal itself is used to track motion. The latter can be implemented directly ${ }^{86}$ or through the use of a short duration "navigator" sequence embedded within the structural or diffusion scan in order to detect and account for motion. Examples of the former include cameras with reflective markers, RF markers, magnetic markers and inertial navigation systems ${ }^{87-89}$. The downside of marker- and sensor-based systems is that the marker or sensor is typically attached to the skin, which may not move rigidly with the brain. Attachment to the upper teeth is more reliable but requires special expertise to set up and assumes that the patient has teeth. An alternative system uses facial geometry itself as the tracked pattern ${ }^{90}$. Camera-based systems require a clear line-of-sight to the subject's head.

An appealing alternative to the hardware and patient preparation involved with external tracking systems is the use of the MR scanner itself to track and remove motion effects ${ }^{91-93}$. If additional navigators are used in the sequence, they may increase scan time but are sometimes inserted in the "dead time" present in many MR sequences during which the scanner is idle while magnetization evolves (either decays or recovers). These methods typically track motion on a slower time-scale than external tracking systems, but require no special setup.

\section{Field Inhomogeneities}


BO (main magnetic field) inhomogeneities/susceptibility regions. Ideally, the main magnetic field (B0 field) should be spatially uniform. Spatial information is encoded by applying linear ramps in the magnitude of the field that vary with position. In practice, the initially uniform field is also modified by the presence of the human subject. Magnetic susceptibility is the material property that describes the amount of magnetization of a material relative to the applied magnetic field. Brain tissue is weakly diamagnetic, dispersing the magnetic field, whereas air, due to the oxygen content, is slightly paramagnetic, concentrating the magnetic field. Some regions of the brain are close to air spaces, such as the inferior frontal region (near the sinuses) and the lateral temporal regions (near the air canals). Shimming is the process of applying additional spatially tailored magnetic fields to compensate for the variation in the magnetic field in these regions. Since the fields generated by the shim coils are fairly smooth, they cannot compensate fully for field inhomogeneity in regions of sharply changing susceptibility. Figure 4 shows how the remaining inhomogeneity results in geometric distortion in the image. In non-EPI acquisitions (such as T1w and T2w), the distortion is in the readout direction (whereas in EPI acquisitions (such as in diffusion MRI) it is along the phase encode direction). As a result of these geometric distortions, the cortex is shown to be compressed or stretched depending on the polarity of the readout (phase-encode for EPI) gradient or equivalently the sign of the field offset. The magnitude of the distortion scales inversely with the readout bandwidth (echo spacing in EPI).

Gradient nonlinearities. Designing a gradient system with high gradient strength and high slew rate, while maximizing spatial linearity and minimizing eddy currents, is an engineering challenge. Linearity is always compromised to some extent. Gradient nonlinearity results in geometric distortion of the images and is the same for all imaging sequences. Since nonlinearity is part of the gradient system design, it does not vary with the patient and, given detailed information about the gradient design, can be corrected on the scanner or offline ${ }^{94}$. Chemical/fat shift. Magnetic spins precess at a rate proportional to the magnetic field that they experience, and the precession frequency encodes the position of the tissue containing the spins. It is most commonly the hydrogen nuclei (protons) in water that are imaged in biological tissue. Water protons precess at a higher frequency than protons in lipids. This difference in precession frequency is called the chemical shift. Since the scanner is tuned to hydrogen nuclei in water, hydrogen nuclei in lipids resonate at the "wrong" frequency, i.e. the frequency of the received signal does not correctly reflect the spatial origin of the source. The result is that the fat signal is shifted in the image relative to the water signal. Just as in regions of B0 field inhomogeneity, the fat shift is inversely proportional to the readout bandwidth. With lower bandwidths, orbital fat or scalp fat may shift sufficiently to overlap with cortex, confounding morphometry studies. The fat signal may be ameliorated through the use of narrowband or composite "water excitation" RF pulses that excite only the water spins and not the fat spins, or through the use of saturation pulses that suppress the fat signal.

$R F(B 1)$ inhomogeneities. Radio frequency (RF) pulses provide the energy that perturbs the spins, which then emit this energy during the relaxation process. The energy experienced by an ensemble of spins is expressed as the "flip angle", because it describes the resulting magnetization in terms of a vector with transverse and longitudinal components (perpendicular and coaxial with the B0 field, respectively). Ideally all the spins within the receive coil should experience the same transmit field $(\mathrm{B} 1+)$ during an RF pulse. In practice, the $\mathrm{B} 1+$ field is not perfectly homogeneous, and the inhomogeneity is exacerbated at higher field strengths where 
the RF wavelength is closer to the scale of the body region being imaged ${ }^{95}$. In the head, this effect leads to brightening of the center of the image. This corresponds to varying flip angle across the head. Note that the result is not simply a scaling of the image intensity, but rather a variation of contrast across the image if multiple tissue types are present. Therefore a simple multiplicative intensity normalization cannot completely correct this effect. One approach is to map relaxation parameters, taking into account the $\mathrm{B} 1+$ map $^{96}$. Achieving a uniform $\mathrm{B} 1+$ field in the first place is ideal, and B1+ uniformity can be improved with parallel transmit methods, where the contributions of multiple transmit coils are combined to achieve a uniform resultant field $^{97}$. Since the combination is phase sensitive and interference can be constructive, great care is necessary to avoid creating RF energy "hot spots".

Once transmitted, RF energy is subsequently emitted from the imaged object during the spin relaxation process. This energy is captured by the receive coils as measured signal and is used to form an image. Multiple receive elements enhance the SNR of the received signal and enable acceleration of image acquisition. However, multichannel receive coils result in an inhomogeneous RF receive field (B1-). This inhomogeneity results in a scaling of the image intensity and can be corrected with a B1 receive map. Optimally the correction should be done by weighting the contributions from each coil element with the coil sensitivity profile before forming the combined image ${ }^{98}$.

\section{Dura}

The human brain and spinal cord are enveloped by three membranes known collectively as the meninges. Of these three - the dura mater, the arachnoid mater and the pia mater - it is the thick dura that can corrupt estimates of cortical volume and thickness. While the dura has MR characteristics that are quite different than gray matter, by unfortunate coincidence the T1/T2/PD of the dura results in it being close to isointense with cortical gray matter in many typical T1-weighted structural imaging sequences. This fact combined with the close proximity or even physical adjacency of the dura to the cortex make it difficult or impossible to avoid having the dura contaminate estimates of cortical thickness and volume from only a T1weighted MRI. Examining Figure 5 one can see many locations where dura is close enough to cortical gray matter that it can be difficult or impossible to resolve the tissue between them and avoid labeling the dura as gray matter. This can lead to substantial overestimation of cortical thickness volume, and more importantly, significant overestimation of atrophy rates when the retraction of the gray matter in neurodegenerative diseases allows the dura to be differentiated from cortex, while it is not differentiated in healthy controls.

Fortuitously, dura has short magnetic decay properties T2 and T2*, and thus even a modest increase in echo time can allow it to be visually distinguished from adjacent gray matter. Unfortunately simply lengthening TE is rarely an optimal strategy for a T1-weighted scan as gray/white contrast reduces monotonically with increasing TE. A better approach is to either use multi-echo acquisitions ${ }^{99}$ where T2* information is encoded in the multiple echoes, or to acquire a bandwidth/readout/geometry matched T2-weighted scan in which the dura will be significantly darker than the cortex. 


\section{Spatial normalization and image processing}

After acquisition is complete, image processing pipelines both extract key metrics as well as identify corresponding spatial mappings between subjects, as described above and illustrated in figures 2 and 3 . While accumulation of computational errors adds noise, it is in the identification of spatial homology that problems can in particular arise. At coarse spatial scales, as well as usually subcortical regions, correspondance between subjects is readily identified and computed. The complex folding patterns of the cerebral cortex and (with sufficient resolution) the cerebellar cortex present a more fundamental problem, in that there is no 1:1 homology between any two cortices. The sulcal patterns vary from cortex to cortex with, to give just one example, with some subjects having a single branched collateral sulcus in the medial temporal lobes, about one third having two branches to the sulcus, and about one fifth of subjects featuring an interrupted sulcus ${ }^{100}$. The branching pattern is furthermore related to volumes of surrounding regions ${ }^{100}$. When such diverging branching patterns exist between subjects - and they exist across many cortical sulci - then it is usually not clear what the correct point to point matching between subjects is, nevermind creating a correct computational algorithm. This is particularly a problem with volumetric registrations, and therefore $\mathrm{VBM}^{101}$.

There are multiple approaches to addressing misregistration problems. The first is to acknowledge that registration issues present one form of signal ${ }^{102}$, i.e., a group difference in grey matter density might be due to differential cortical folding rather than true grey matter amounts, and thus a finding in VBM provides an indication of where to look in more detail at anatomical alterations. Second, explicitly aligning cortical folding patterns significantly improves localization. The best illustration for this improvement is in the cross-subject correspondence of Broca's areas (cortical subdivisions based on neuron types and distributions invisible on MRI) after either volumetric or surface based registration, with the surface based algorithm considerably improving co-localization ${ }^{103}$. Even surface based registrations cannot completely account for differential folding patterns (where the true solution is not even known), and thus the third approach is explicitly map sulcal shapes. The best of known suite of algorithms for sulcal identification and study is in BrainVISA ${ }^{104}$. Here sizes, location, branching patterns, etc., can be studied and compared across groups or other metrics of interest. Ultimately, these methods are complementary, and as with acquisition artefacts, understanding the role of possible confounds on final outcomes is key in best advancing science using structural brain imaging.

\section{Structural imaging: now and in the future}

Advances in ultra-high field strengths (e.g. 7T) offer a route for increased baseline signal (and therefore SNR). This comes at other costs, particular for acquisitions that are affected by shorter T2 relaxation times, worse radio-frequency field homogeneity, greater radio-frequency power deposition and increased magnetic susceptibility and potential for distortions ${ }^{95}$. In particular, methods such as diffusion and fast acquisitions are challenging to implement robustly at higher field. Nevertheless, establishing the right balance between all these factors allows data of very high quality and high spatial resolution ${ }^{105,106}$. Ultra-high field can also have transformative impact for certain modalities that carry limited information at lower fields. Quantitative susceptibility mapping is an example, with increased contrast at $7 \mathrm{~T}$ enabling whole-brain quantitative 
susceptibility mapping ${ }^{107}$ and higher spatial resolution providing iron deposition imaging even in small subcortical nuclei ${ }^{108}$.

At the same time, multi-channel receive coil arrays allow for higher SNR and simultaneous acquisition of more than one slice. The volume extent to be imaged can be separated into a set of equally spaced slices, each driving high signal in a unique subset of coil elements. The slices can then be acquired simultaneously and the spatial sensitivity of each coil element can be used to separate the signal from different slices. Such simultaneous multislice (or multiband) acquisitions ${ }^{109,110}$ reduce the time required to scan brain volumes and currently permit 2-5-fold acceleration of dMRI scan time ${ }^{111}$, translating to higher spatial and/or angular resolution and/or SNR per unit time. Multiband acquisitions have been key in improving data quality in different projects (and contexts), such as the adult Human Connectome Project ${ }^{6}$, UK Biobank $^{5}$ and the developing Human Connectome Project ${ }^{112}$.

Modern clinical scanners are equipped with gradient systems that can deliver greater spatial magnetic fields gradients $G_{\max }(\sim 80 \mathrm{mT} / \mathrm{m}$ vs $40 \mathrm{mT} / \mathrm{m})$. In diffusion imaging, higher gradient strength leads to greater signal contrast in terms of the signal change for a given displacement of water due to diffusion. In addition, experiments with precise control over the gradient strength and duration (collectively pooled in the parameter $q$ ) can permit measurement of small differences in diffusion displacement that may enable estimation of specific microstructural features of tissue. Furthermore, tight head-gradient sets allow fast switching of the gradients (i.e. faster "slew rates") compared to whole-body gradient systems. These features collectively allow shorter and stronger gradient pulses for diffusion preparation, which are beneficial in a number of ways, including higher SNR/spatial resolution/angular contrast and more accurate q-space measurements that technically require infinitely short gradients. Human MR systems have been recently developed with ultra-high $G_{\max }$, such as the Connectom Skyras with 100 and $300 \mathrm{mT} / \mathrm{m}^{95,113}$, allowing very high data quality ${ }^{114,115}$. Such hardware capabilities have been very beneficial in mapping microstructure in animals using small-bore systems, and the potential to translate these advantages into in human studies has recently been demonstrated $^{116,117}$.

\section{The Future}

Advances in acquisition, image processing, and modelling will assuredly keep structural imaging at the forefront of understanding brain-behavior relations and how they are altered in development, ageing, and disease.

A primary focus for diffusion MRI research is in microstructural models that go beyond tensor-derived metrics, such as FA, which are inherently non-specific. For instance, a reduction in FA has been associated with loss of structure/ "integrity", while an increase in FA can be indicative of degeneration ${ }^{118}$. In regions with complex fibre patterns, selective degeneration of one fibre population can lead to an apparent increase in structural coherence; what is left looks macroscopically more "organised".

More complex biophysical models along with sophisticated measurements can more precisely explain the measured signal ${ }^{119}$ and aid interpretation by providing specific markers of microstructure changes ${ }^{120-122}$. For example, the diffusion signal measured at a range of $q$ values 
(gradient areas) exhibits a characteristic diffraction pattern in which zero crossings indicate the size of restricted compartments ${ }^{123}$. Estimation of microscopic features, such as compartment shape and size, using this approach have been demonstrated on non-biological model systems using high-performance scanners ${ }^{124}$. This approach might ultimately enable estimation of mean axon diameters from measurements made perpendicular to a perfectly coherent white matter fiber bundle. In practice, however, such a diffraction pattern cannot be measured with the standard (single-pulse) diffusion sequences or using current hardware available on human scanners. Moreover, these models typically neglect the heterogeneity of fibres within a voxel, which can drive similar signal properties to that of compartmental shape. A recent method ${ }^{125}$ aims to estimate "per-axon" diffusivities and anisotropy ("microscopic anisotropy"), which are inherently free from orientation homogeneity/heterogeneity assumptions and focus solely on microscopic features of interest. Variations in the gradients used for diffusion encoding, primarily involving double diffusion encoding approaches such as double-pulse diffusion sensitisation or oscillating gradients, offer alternative approaches for estimating compartment shape and size, as "diffraction" measurements of zero-crossings are robust to heterogeneities of the imaged system $^{124,126,127}$. Such sequences are also promising to better characterise restricted diffusion and probe anisotropy within a bulk isotropic voxel (e.g. ${ }^{128-130}$ ) (see Box1, bottom right figure panel) and even capture diffusional water exchange, a cell membrane permeability dependent parameter ${ }^{131}$. While these various methods have demonstrated remarkable potential in model systems, translation into in-vivo human imaging remains limited by the hardware and measurement techniques available on human MRI scanners, though that is rapidly improving (e.g. ${ }^{125,131}$ ) and will likely provide exciting breakthroughs in the future. These improvements in diffusion imaging techniques will furthermore assist with better tractography, thereby enabling improved localization of the signals origins.

We face an exciting future for the study of neuroanatomy as methods advance, artifacts are understood and their impacts reduced. Our understanding of how the brain changes, be it across development or ageing, in response to novel environments or cognitive challenges, or in disease, is still rudimentary. Combining estimates of volumes, thickness, etc. from high resolution anatomical images with multiple microstructure modalities, including double diffusion encoding approaches, magnetization transfer and susceptibility weighted imaging and quantitative T1 and T2 mapping, is especially promising. Combining in-vivo human imaging with experimental models, with all their genetic and molecular tools, will also provide novel insights. Ultimately, we will come closer and closer to not just mapping what is happening where, but to understanding the underlying molecular and cellular bases of our signals.

Also, brain structure provides a common framework to unify multiple neuroscience investigations. Recent years have seen a proliferation of spatially comprehensive and publicly available maps of human brain organization that are all anatomically-grounded, but together encompass a vast array of phenotypic dimensions. Salient examples include spatial maps of gene expression (e.g. Allen Brain Atlas http://brain-map.org), cytoarchitectonics (https://www.jubrain.fz-juelich.de/apps/cytoviewer/cytoviewer-main.php), and cognitive associations (http://neurosynth.org). The diverse modalities represented by these maps emphasizes the fact that structural neuroimaging provides just one of many ways of modelling 
the brain, but also reinforces the fundamental importance of anatomy as the common spatial framework within which all other phenotypic properties of the brain are embedded ${ }^{132}$. This broad point is well illustrated by several relatively recent studies that use structural neuroimaging data to calculate physical distances between different brain regions, and then demonstrate that these distance-based representations of the brain can predict spatial patterns of functional connectivity, metabolism, gene expression and cognitive specialization within the human brain ${ }^{133,134}$. Thus, the in vivo measures of brain organization provided by structural neuroimaging are not only highly informative and discriminative phenotypes in their own right, but describe the basic anatomical scaffold within which our brains evolve, develop and operate.

\section{Figures}

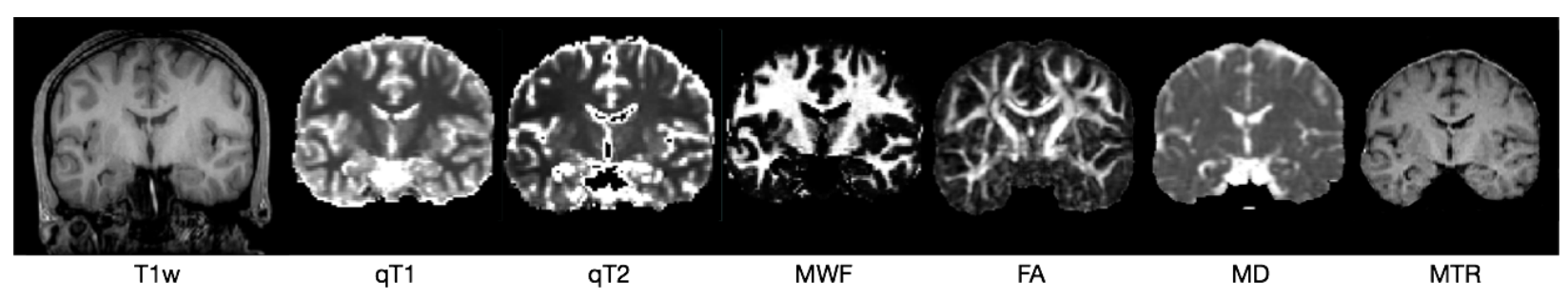

Figure 1: Coronal slices of multi-modal images of brain structure acquired in members of a birth cohort when they reached 20 years of age (funded by R01MH085772-01A1: "Axon, Testosterone and Mental Health during Adolescence", T. Paus). From left to right, they are the T1 weighted ( $\mathrm{T} 1 \mathrm{w})$ image most commonly used for analyzing brain volumes, voxel based morphometry, cortical thickness, etc. Next are the quantitative T1, T2, and myelin water fraction (MWF) maps estimated using the mcDESPOT sequence ${ }^{45}$. Fractional anisotropy (FA) and mean diffusivity (MD) from diffusion imaging and a magnetization transfer ratio (MTR) map finish up the set of images. These data indicate the type of rich information about brain structure that can be obtained from MRI in a single session. 


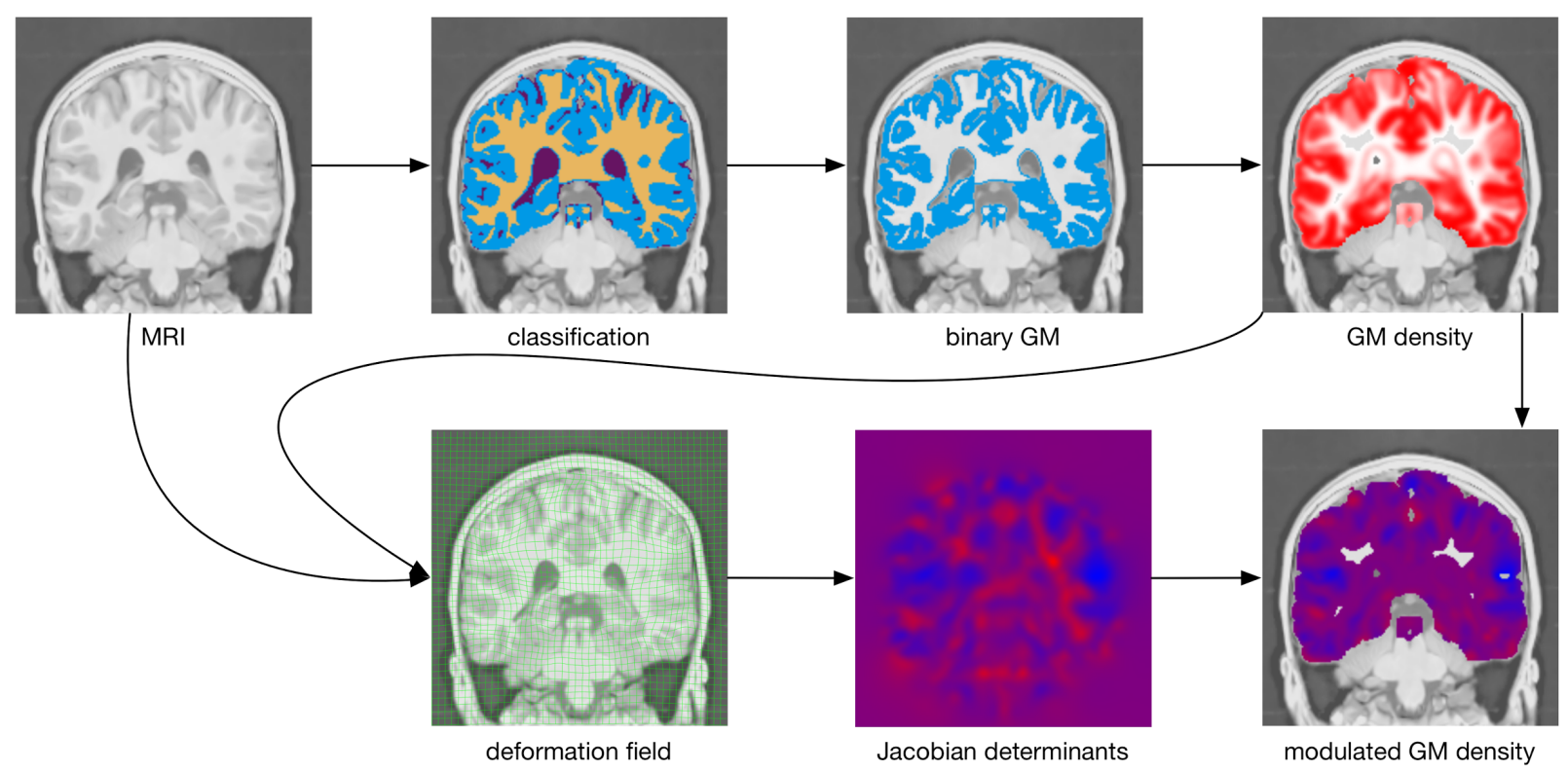

Figure 2: Voxel Based Morphometry (VBM). VBM was the first widely adopted technique for determining alterations in neuroanatomy across sets of subjects. VBM entails classifying the brain (MRI) into white matter, grey matter, cerebrospinal fluid, and background (classification), extracting one of the classified tissues types (binary $G M$ ), then smoothing the extracted tissue type with a Gaussian kernel. The final product is thus an image (GM density), in linearly registered stereotaxic space, with values ranging from 0 to 1 representing the amount of grey matter within a local neighbourhood determined by the blurring kernel ${ }^{8}$. A modification to the basic VBM protocol was proposed in $2001^{11}$, wherein non-linear registration, based on either aligning the T1 weighted MRIs or the GM density maps, is incorporated to provide better spatial alignment. This optimized VBM procedure also combines the non-linear registration (deformation field) with the tissue density map obtained from classic VBM by multiplying (or modulating) the tissue density map by the Jacobian determinant of the non-linear deformation field to produce the modulated GM density map. Thanks to Chris Hammill for his assistance with these figures. 

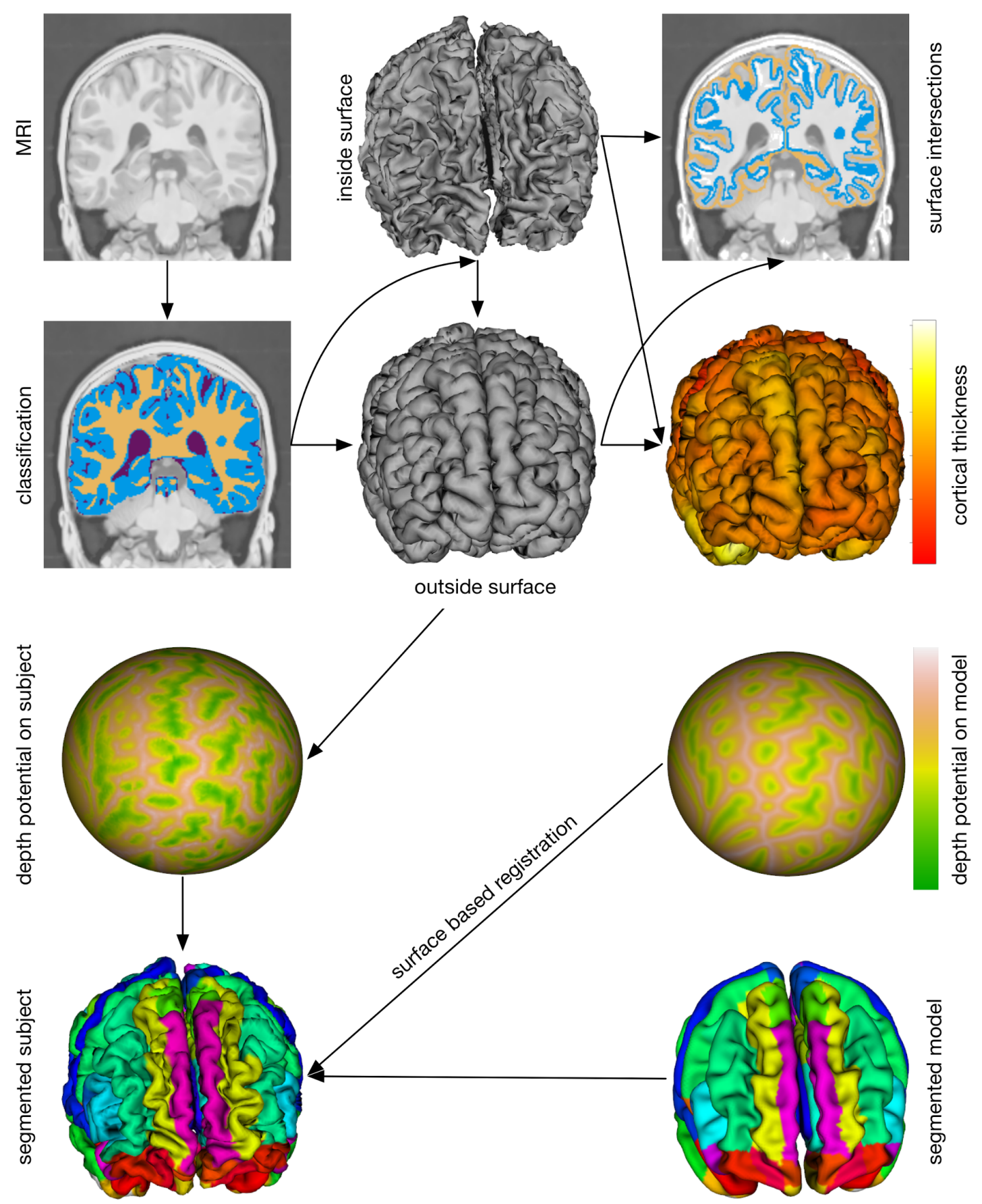

Figure 3: surface based analyses. The inside surface and outside surface of the cortex are extracted based on a mix of tissue classification and deformable model segmentation. Cortical thickness can then be computed based on the distance between the inside surface and outside surface, and surface area computed on either surface (not shown). For the sake of inter-subject statistics as well as to aid in segmenting the cortex into constituent lobes, sulci, and gyri, the curvature (or, alternately, some measure of sulcal depth or depth potential) is computed on the 
surface (depth potential on subject) as well as for a model (depth potential on model). Surface based registration then takes the segmented model and uses it to parcellate the input surface (segmented subject). 


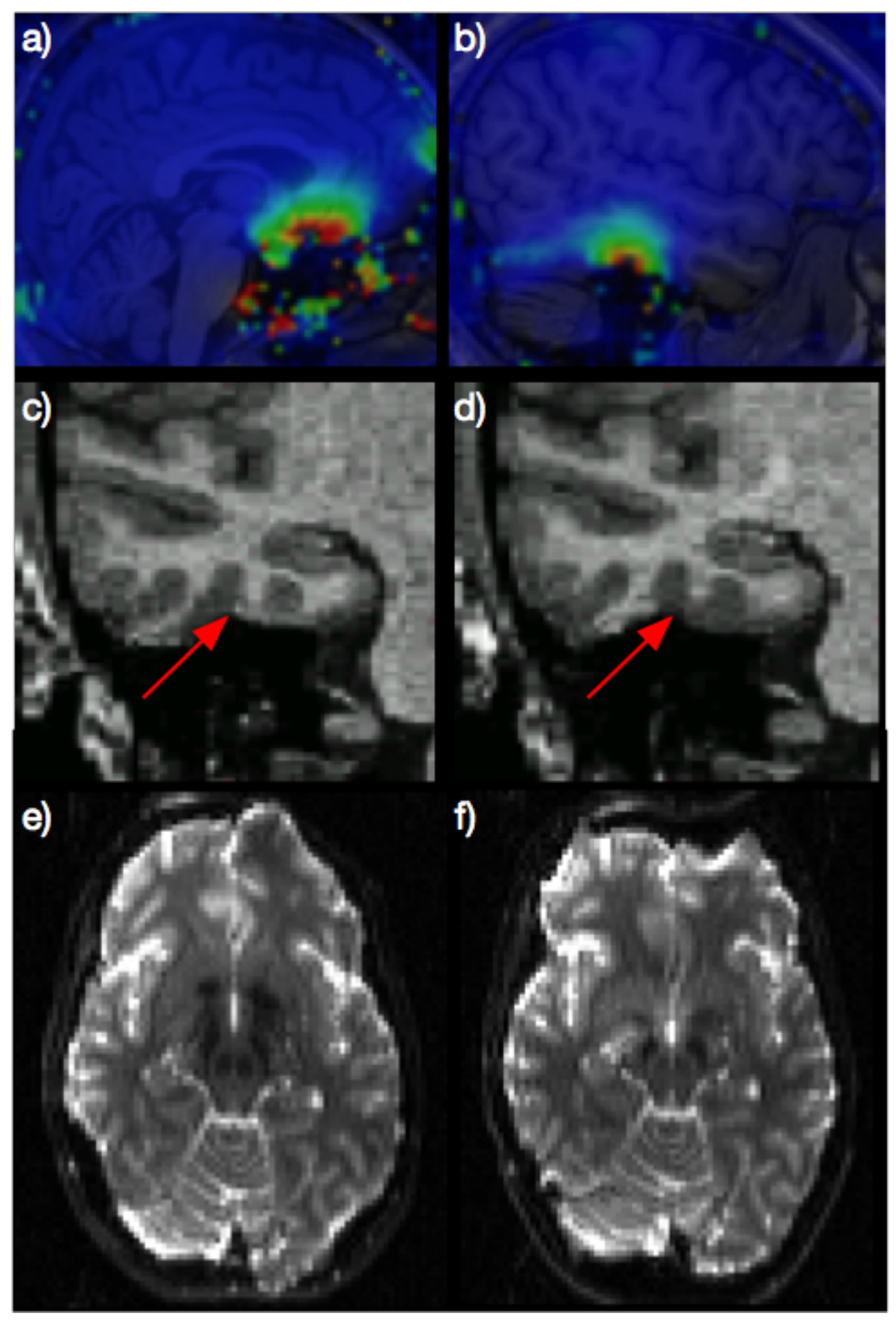

Figure 4. From left to right B0 field maps in orbitofrontal (a) and lateral temporal (b) regions showing large inhomogeneities in the magnetic field. Images in (c) and (d) are FLASH scans in which the polarity of the readout gradient is changed from positive (c) to negative (d), changing the direction of the distortion. As can be seen in the region of the red cursor, the effect of this is 
to have no apparent cortex in image (c), but quite thick cortex (d). The effect is even more dramatic when changing the polarity of the phase encode direction in diffusion images, as seen in (e) and (f). 


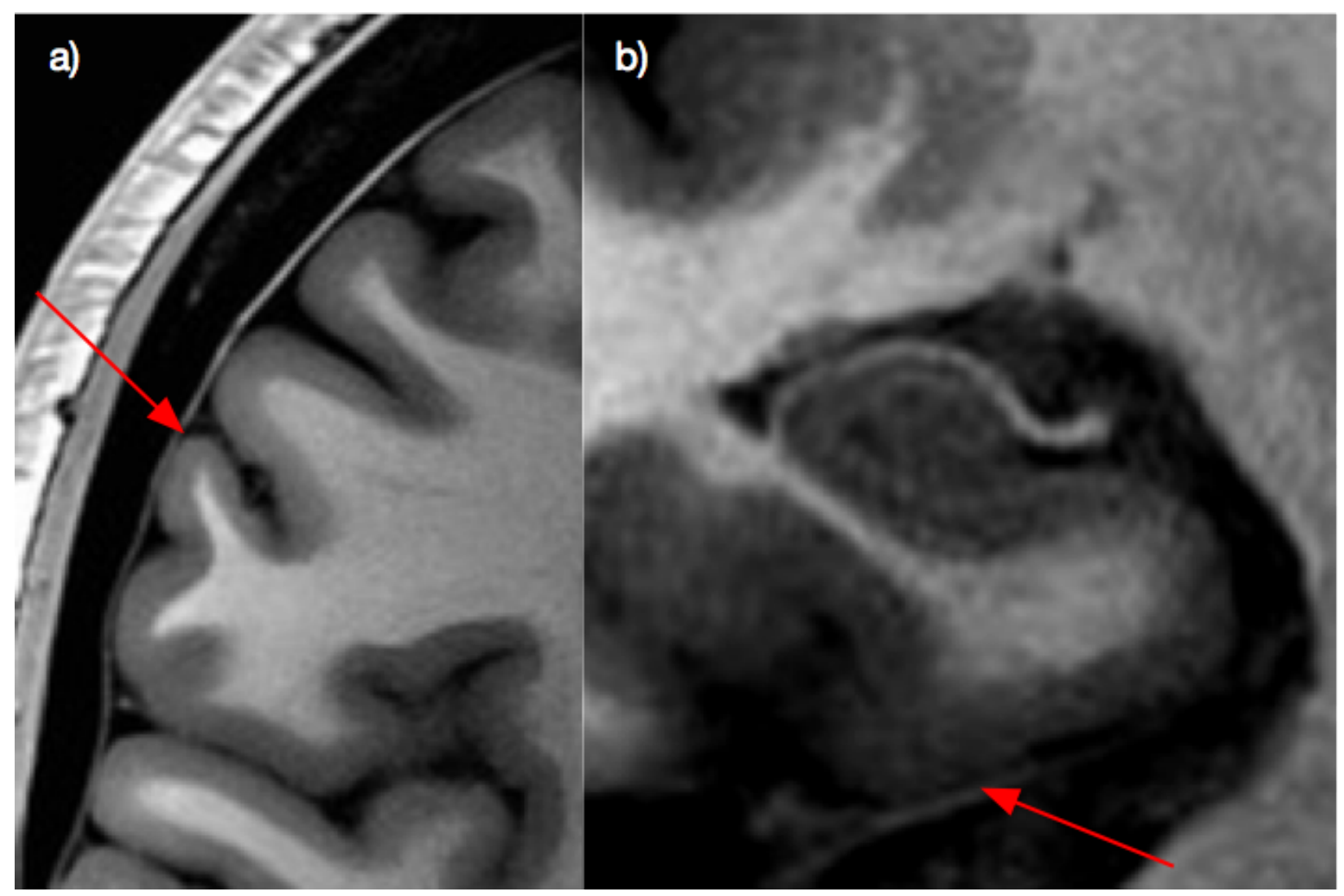

Figure 5. 380um $\times 380 \mathrm{um} \times 1 \mathrm{~mm}$ image (average of 7 scans of 9 minutes each motion corrected $\mathrm{Tl}=900 \mathrm{~ms}$, TR/TE/flip = 2250/4.35/9deg, 3T, 32 channel array) highlighting the dura and its proximity to superior lateral cortex (a) as well as entorhinal (b). Thanks to Larry Wald for providing these images. 


\section{Boxes}

\section{Box 1: advanced diffusion modelling}
A) DTI FA
B) CROSSING
E) "NEURITE"
F) ORIENTATION

FIBRES

DENSITY

DISPERSION

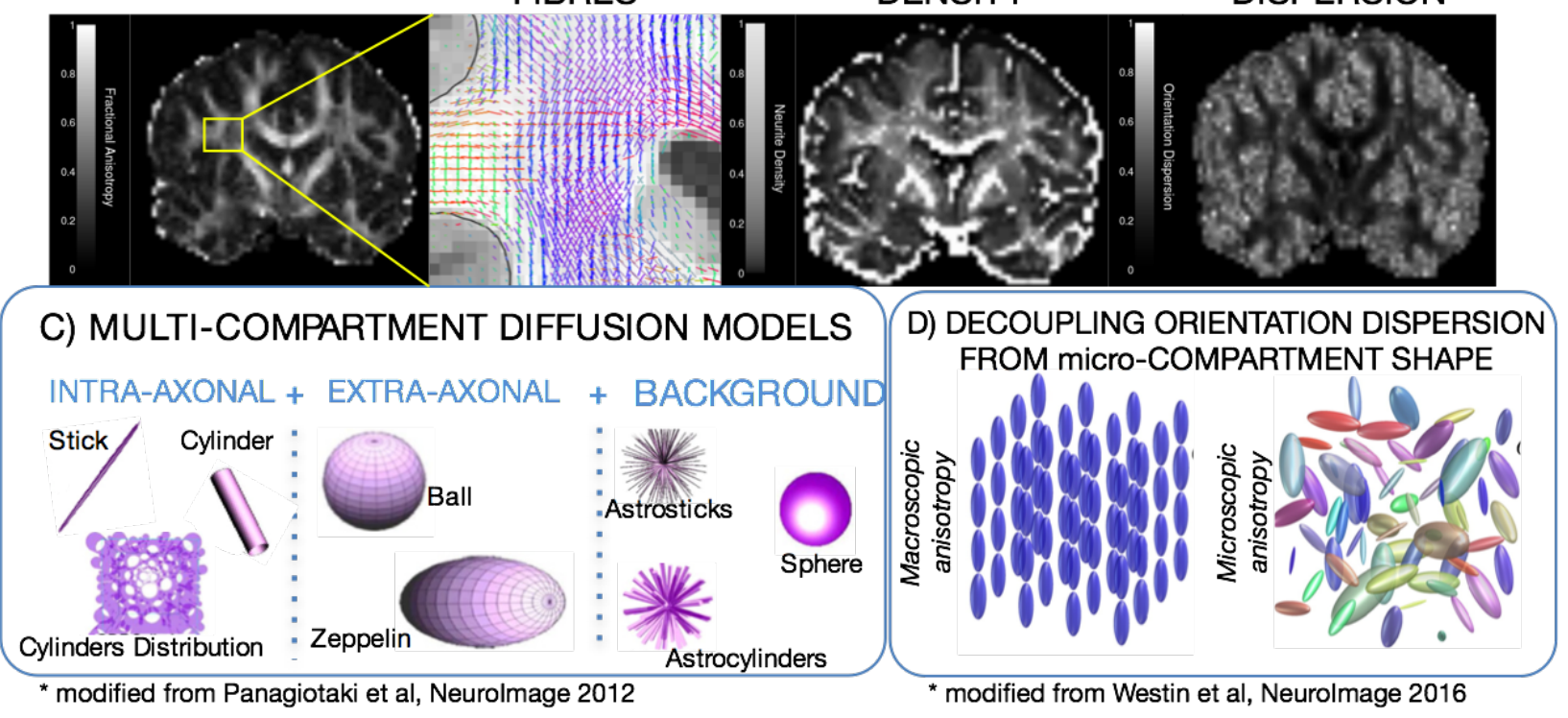

Figure 6

Tensor-based modelling of diffusion MRI data assumes that water molecules disperse randomly according to a Gaussian distribution that is in general anisotropic (i.e. described by an ellipse). The tensor model can provide markers that are sensitive to alterations in tissue microstructure, but tensor-based metrics are not specific to biologically interpretable tissue properties. Furthermore, tensor-derived measures like FA (Fig 6A) reflect geometrical properties (fiber complexity, Fig 6B), biophysical properties (size of fibers and/or fiber density) and aspects of the measurement (diffusion time and/or b-value).

A number of biophysical models have been proposed as an alternative to the tensor to aid interpretation by parameterising the observed signal as a function of intrinsic tissue properties (see ${ }^{128}$ for a review and ${ }^{119}$ for a taxonomy of these models). Early on, the observation of multi-exponential decay of the diffusion signal (particularly at high b-values) ${ }^{135,136}$ motivated multi-compartment models (Fig 6C), where the signal is assumed to arise from non-exchanging cellular environments. For example, early models treated the extra-cellular space as giving rise to hindered diffusion (Gaussian movement but with a reduced diffusion coefficient), and intracellular spaces as having restricted diffusion (which is inherently non-Gaussian) $)^{137}$. Several approaches have expanded on these earlier studies, in particular for modelling white matter. The ball-and-stick model ${ }^{138}$ captured restricted, perfectly anisotropic intra-axonal diffusion (stick) and isotropic extra-axonal diffusion (ball). The composite hindered and restricted water diffusion (CHARMED) model $^{139}$ replaced the sticks with cylindrical fibers of fixed diameter and modeled 
the extra-axonal space using a tensor. AxCaliber ${ }^{140}$ further added a distribution of axon diameters and included a compartment to represent stationary/glial cell water ${ }^{141}$. Modified versions of the above models were used in ${ }^{142}$ to provide a model for white matter diffusion that was relatively simple and yet complex enough to probe axonal density and diameter. Fieremans et al. ${ }^{35}$ also proposed a mapping from non-specific diffusion kurtosis metrics to white matter microstructure features (including axonal water fraction, intra-axonal diffusivity, extra-axonal diffusivities).

The previous models assume homogeneity in fiber orientation. A plethora of methods exist for the estimation of the fiber orientation density function (fODF), which describes the distribution of fiber orientations within each imaging voxel (see ${ }^{143}$ for a review). fODF estimation methods are mostly designed and aimed for tractography, although some microstructure information can be extracted (for instance ${ }^{118,144}$ ). Nevertheless, fiber dispersion is represented explicitly in more recent multi-compartment white matter models (Fig 6D) that relax the assumption of orientation homogeneity $\left(\right.$ e.g. ${ }^{145,146}$ ). Dispersion models are also increasingly being used to make inferences about grey matter compartments that collectively represent neurites, i.e. projections from cell bodies, either dendrites or axons ${ }^{72}$. Recently, the NODDI (neurite orientation dispersion and density imaging) model has enabled similar measures to be estimated from images acquired on commonly available clinical scanners using scan times that are feasible for in-vivo imaging ${ }^{147}$, (Fig 6E-F).

\section{Box 2: population neuroscience}

Population neuroscience integrates epidemiology, genetics and neuroscience to identify influences shaping the human brain from conception onwards ${ }^{5,148,149}$. As we discussed elsewhere ${ }^{150}$, such efforts face three key challenges: (1) an infinite combination of factors influencing the brain from within (genes and their regulation) and the outside (social and physical environment); 2) presence of developmental cascades that carry such influences from one time point to the next (e.g., prenatal to postnatal), from one organ to another (e.g., cardiometabolic to brain), and from one level of organization to a different one (e.g., behavior to gene regulation and vice versa); and (3) structural and functional complexity of the human brain.

These challenges can be met by conducting studies in large samples of participants drawn from the general population and evaluated with state-of-the-art tools for assessing (a) genes and their regulation; (b) external and internal environments; and (c) brain properties; all done in an integrative fashion and across lifespan. Unlike clinical (case-control) studies, population neuroscience does not focus on patients. An ideal (i.e., representative) sample includes a mix of healthy individuals, individuals in pre-clinical stages of a disease and those with a fully blown disorder, with numbers corresponding to the population prevalence of different conditions (and their antecedents) at a given age. Broad sampling of environments and genomes is essential for identifying the key influences shaping the brain capacities (and health) under different circumstances.

The usefulness of various datasets for answering certain questions is determined not only by what had been acquired but also by who participated in the study. Ascertainment (to ascertain $\sim$ to discover) refers to the way we identify individuals for the purpose of recruiting 
them into a given study. Ascertainment bias refers to a sampling (or selection) bias. Such a bias is more likely to occur in case-control studies (e.g., patients with a certain disease vs. healthy controls) but may be present also in population-based studies recruiting participants in the community. In all cases, it is crucial to document (and report) recruitment strategies, target populations and, if possible, response rates and characteristics of those who declined to participate (or who might have been excluded by investigators).

To date, most of the population-neuroscience work has been done in high-income countries and carried out in the context of observational studies. This is the case for the great majority of sites participating in the Enhancing Neurolmaging Genetics through Meta-Analysis (ENIGMA) consortium ${ }^{151}$ and the Cohorts for Heart and Aging Research in Genomic Epidemiology (CHARGE) consortium ${ }^{152}$. These two consortia brought together various studies employing brain imaging and genetics in disease-oriented research carried out mostly in North America, Europe, Australia and Japan.

Many of the population-based studies have combined MR imaging with detailed assessments of various environments, including internal milieu (e.g., plasma levels of hormones, lipids, micronutrients), physical (e.g., air pollution) and social environment (e.g., social structure of the family and neighborhood), as well as previous experiences (e.g., stressful life events). Assessing "external" environments is challenging. Asking a series of questions using a standard survey is the most common way for collecting information about the individual's physical and social environment (e.g., https://www.phenxtoolkit.org/index.php). One can also use aggregate data (collected by local, state or federal governments) to characterize physical and social environments in which an individual spends most of his/her time (e.g., home, workplace). This can be done using Geographic Information Systems that allows one to map data in space and time, providing multiple layers of information about the individual's physical and social environment at a given time point (e.g., http://www.esri.com/). Furthermore, the emergence of tools such as Google Street View and social media, including Twitter and Facebook, (see Odgers et al. ${ }^{153}$ for an example) provides new opportunities for characterizing physical and social environment at an aggregate level.

In addition to being scanned and assessed in a number of domains (e.g., cognition, mental and cardio-metabolic health), cohort participants provide a blood sample that can be used subsequently in genome-wide genomic, transcriptomic and epigenomic analyses. It is of note that proper acquisition, processing and storage of blood and its derivates (e.g., plasma, serum, blood cells) provide a rich source of biological material to be used with technologies often unavailable when a study started (e.g., whole-genome sequencing, derivation of inducible Pluripotent Stem Cells). To some extent, this is also true about imaging data: new imageanalysis algorithms may be used to derive new phenotypes from old images.

Let us finish this section with one example of integrating system-level and molecular phenotypes derived, respectively, from in vivo (MRI) and ex vivo (gene expression) studies. For many years, multi-modal integration of various structural and functional features of the human brain has been enabled by the use of a common reference system ${ }^{154}$. This mapping principle has been used to bring gene-expression data contained in the Allen Brain Atlas into a FreeSurfer-based anatomical parcellation of the cerebral cortex ${ }^{155}$. This allowed us, for example, to show that regional differences in cortical thickness associated with early cannabis use follow a regional gradient in the expression of $C N R 1^{156}$. 


\section{Box 3: developmental structural neuroimaging}

Our first major longitudinal insight into the dynamics of in vivo human brain development came from a structural neuroimaging study ${ }^{157}$, which revealed that the maturational trajectory of human gray matter volume follows a curvilinear "inverted-U", rather than showing a linear progression to adult values. Since this seminal study there has been a steep climb in the number of large-scale longitudinal structural neuroimaging datasets ${ }^{158}$ and associated research reports detailing the spatiotemporal heterogeneity of neuroanatomical maturation within the brain $^{16,159}$, and charting how the dynamics of structural brain development can vary as a function of genetic ${ }^{160}$, environmental ${ }^{19}$, demographic (e.g. biological sex ${ }^{159}$ ), cognitive ${ }^{161}$ and clinical ${ }^{162}$ factors. Several special opportunities and challenges of structural neuroimaging are brought to the fore by developmental applications, and deserve special mention.

First, advances in automated image analysis have meant that a vast number of morphological estimates can now be extracted from any given structural neuroimaging scan. For example, modern methods for cortical morphometry measure cortical thickness, surface area, volume, curvature and sulcal depth relative to the brain hull at tens of thousands of points across the cortical mantle in a single scan. It has become clear that these diverse metrics follow distinct developmental trajectories in health ${ }^{163}$, which reflect non-overlapping sets of genetic and environmental influences ${ }^{18}$, but can be inter-related in a spatiotemporally specific manner ${ }^{164}$. These normative observations carry major consequences for the optimal design of structural neuroimaging analysis in clinical populations, because conclusions regarding the presence and regional distribution of cortical abnormalities in a given genetic disorder can vary greatly across different morphometric features ${ }^{159}$.

Second, any effort to localize anatomical changes that segregate with a demographic or clinical variable of interest needs to be balanced against the recognition that regional variations in brain size/shape are not independent from each other or from variations in overall brain size. From a statistical perspective, this observation raises the question about how to best "account for" total brain size when analyzing a region of interest (ROI) - which has traditionally been addressed by either proportionalizing ROI measures, or including total brain volume as a linear covariate when modelling variation in ROI anatomy. However, a number of recent studies ${ }^{165,166}$ have underlined that relationships between ROI size and overall brain size are often profoundly non-linear, and shown how the inability of traditional methods to control for these non-linearities can lead to false inferences regarding the presence and location of regional brain changes. The importance of using an allometric framework in analysis of morphometric data is especially pronounced when studying groups that differ markedly in overall brain size due to demographic (e.g males vs. females) or clinical (e.g. neurogenetic syndromes vs. controls) variables ${ }^{166}$.

Third - in addition to raising important analytic challenges - population-level patterns of neuroanatomical covariation represent a special window into brain organization that is uniquely provided by structural neuroimaging data and offers new ways of detecting brain changes secondary to developmental or disease-related processes. Thus, patterns of structural 
covariance are known to vary over childhood and adolescence ${ }^{50}$, track inter-individual differences in cognitive ability ${ }^{167}$, be altered by disease processes ${ }^{168}$, and recapitulate patterns of maturational coupling ${ }^{163}$, inter-regional connectivity and coordinated functional activation within the human brain ${ }^{169,170}$. Crucially, normative patterns of anatomical covariation in the human brain appear to constrain the spatial distribution of disease effects ${ }^{168,171}$, which advances our ability to assay and interpret structural neuroimaging phenotypes in clinical populations.

Fourth, advances in image acquisition and image processing allow us to probe very early brain development. 3D in-utero imaging has to cope with large rotations of the fetus during the scans. By taking multiple stacks of $2 \mathrm{D}$ slices, where each slices is acquired fast enough to negate most motion, and then accounting for motion between slices, a 3D image can be reconstructed ${ }^{172}$. Rapid myelination and similar maturational changes in the developing brain furthermore result in MR contrast changes (i.e. T1 and T2) throughout the first 24 months of life. Image processing algorithms thus have to adapt to these changing contrasts ${ }^{173}$. Lastly, preterm and very preterm born subjects are also providing new insights into early brain development without the constraints of in-utero acquisitions ${ }^{174}$. These populations can furthermore capture effects of altered environments on early brain development ${ }^{175}$.

\section{References Cited.}

1. Zilles, K. \& Amunts, K. Centenary of Brodmann's map - conception and fate. Nat. Rev. Neurosci. 11, 139-145 (2010).

2. Gowland, P. A. \& Stevenson, V. L. in Quantitative MRI of the Brain 111-141 (John Wiley \& Sons, Ltd, 2003).

3. Bottomley, P. A., Hardy, C. J., Argersinger, R. E. \& Allen-Moore, G. A review of 1H nuclear magnetic resonance relaxation in pathology: are T1 and T2 diagnostic? Med. Phys. 14, 137 (1987).

4. Boulby, P. A. \& Rugg-Gunn, F. T2: the transverse relaxation time. Quantitative MRI of the brain. Wiley, Chichester 143-202 (2003).

5. Miller, K. L. et al. Multimodal population brain imaging in the UK Biobank prospective epidemiological study. Nat. Neurosci. (2016). doi:10.1038/nn.4393

6. Glasser, M. F. et al. The Human Connectome Project's neuroimaging approach. Nat. 
Neurosci. 19, 1175-1187 (2016).

7. Chakravarty, M. M. et al. Performing label-fusion-based segmentation using multiple automatically generated templates. Hum. Brain Mapp. 34, 2635-2654 (2013).

8. Ashburner, J. \& Friston, K. J. Voxel-Based Morphometry-The Methods. Neuroimage 11, 805-821 (2000).

9. Cao, J. \& Worsley, K. J. The detection of local shape changes via the geometry of Hotelling's T^2 fields. Ann. Stat. 27, 925-942 (1999).

10. Chung, M. K. et al. A unified statistical approach to deformation-based morphometry. Neuroimage 14, 595-606 (2001).

11. Good, C. D. et al. A voxel-based morphometric study of ageing in 465 normal adult human brains. Neuroimage 14, 21-36 (2001).

12. Dale, A. M., Fischl, B. \& Sereno, M. I. Cortical surface-based analysis. I. Segmentation and surface reconstruction. Neuroimage 9, 179-194 (1999).

13. Fischl, B., Sereno, M. I. \& Dale, A. M. Cortical surface-based analysis. II: Inflation, flattening, and a surface-based coordinate system. Neuroimage 9, 195-207 (1999).

14. Kim, J. S. et al. Automated 3-D extraction and evaluation of the inner and outer cortical surfaces using a Laplacian map and partial volume effect classification. Neuroimage 27, 210-221 (2005).

15. Ducharme, S. et al. Trajectories of cortical thickness maturation in normal brain development--The importance of quality control procedures. Neuroimage 125, 267-279 (2016).

16. Amlien, I. K. et al. Organizing Principles of Human Cortical Development--Thickness and Area from 4 to 30 Years: Insights from Comparative Primate Neuroanatomy. Cereb. Cortex 26, 257-267 (2016).

17. Raznahan, A. et al. Globally Divergent but Locally Convergent X-and Y-Chromosome Influences on Cortical Development. Cereb. Cortex 26, 70-79 (2016). 
18. Chen, C.-H. et al. Genetic topography of brain morphology. Proc. Natl. Acad. Sci. U. S. A. 110, 17089-17094 (2013).

19. Raznahan, A., Greenstein, D., Lee, N. R., Clasen, L. S. \& Giedd, J. N. Prenatal growth in humans and postnatal brain maturation into late adolescence. Proc. Natl. Acad. Sci. U. S. A. 109, 11366-11371 (2012).

20. Schmaal, L. et al. Cortical abnormalities in adults and adolescents with major depression based on brain scans from 20 cohorts worldwide in the ENIGMA Major Depressive Disorder Working Group. Mol. Psychiatry (2016). doi:10.1038/mp.2016.60

21. Smith, E. et al. Cortical thickness change in autism during early childhood. Hum. Brain Mapp. 37, 2616-2629 (2016).

22. Lerch, J. P. \& Evans, A. C. Cortical thickness analysis examined through power analysis and a population simulation. Neuroimage 24, 163-173 (2005).

23. Jbabdi, S., Sotiropoulos, S. N., Haber, S. N., Van Essen, D. C. \& Behrens, T. E. Measuring macroscopic brain connections in vivo. Nat. Neurosci. 18, 1546-1555 (2015).

24. Tardif, C. L., Collins, D. L. \& Pike, G. B. Sensitivity of voxel-based morphometry analysis to choice of imaging protocol at 3 T. Neuroimage 44, 827-838 (2009).

25. Tardif, C. L., Collins, D. L. \& Pike, G. B. Regional impact of field strength on voxel-based morphometry results. Hum. Brain Mapp. 31, 943-957 (2010).

26. Lüsebrink, F., Wollrab, A. \& Speck, O. Cortical thickness determination of the human brain using high resolution 3T and 7T MRI data. Neuroimage 70, 122-131 (2013).

27. Scholtens, L. H., de Reus, M. A. \& van den Heuvel, M. P. Linking contemporary high resolution magnetic resonance imaging to the von Economo legacy: A study on the comparison of MRI cortical thickness and histological measurements of cortical structure. Hum. Brain Mapp. 36, 3038-3046 (2015).

28. Basser, P. J., Mattiello, J. \& LeBihan, D. MR diffusion tensor spectroscopy and imaging. Biophys. J. 66, 259-267 (1994). 
29. Pierpaoli, C. \& Basser, P. J. Toward a quantitative assessment of diffusion anisotropy. Magn. Reson. Med. 36, 893-906 (1996).

30. Simon, T. J. et al. Volumetric, connective, and morphologic changes in the brains of children with chromosome 22q11.2 deletion syndrome: an integrative study. Neuroimage 25, 169-180 (2005).

31. Voineskos, A. N. et al. Quantitative examination of a novel clustering method using magnetic resonance diffusion tensor tractography. Neuroimage 45, 370-376 (2009).

32. Smith, S. M. et al. Tract-based spatial statistics: voxelwise analysis of multi-subject diffusion data. Neuroimage 31, 1487-1505 (2006).

33. Yushkevich, P. A., Zhang, H., Simon, T. J. \& Gee, J. C. Structure-specific statistical mapping of white matter tracts. Neuroimage 41, 448-461 (2008).

34. Jensen, J. H. \& Helpern, J. A. MRI quantification of non-Gaussian water diffusion by kurtosis analysis. NMR Biomed. 23, 698-710 (2010).

35. Fieremans, E., Jensen, J. H. \& Helpern, J. A. White matter characterization with diffusional kurtosis imaging. Neuroimage 58, 177-188 (2011).

36. McGowan, J. C. The physical basis of magnetization transfer imaging. Neurology 53, S3-7 (1999).

37. Kucharczyk, W., Macdonald, P. M., Stanisz, G. J. \& Henkelman, R. M. Relaxivity and magnetization transfer of white matter lipids at MR imaging: importance of cerebrosides and pH. Radiology 192, 521-529 (1994).

38. Laule, C. et al. Magnetic resonance imaging of myelin. Neurotherapeutics 4, 460-484 (2007).

39. Pike, G. B. Pulsed magnetization transfer contrast in gradient echo imaging: a two-pool analytic description of signal response. Magn. Reson. Med. 36, 95-103 (1996).

40. Ward, K. M., Aletras, A. H. \& Balaban, R. S. A new class of contrast agents for MRI based on proton chemical exchange dependent saturation transfer (CEST). J. Magn. Reson. 143, 
$79-87$ (2000).

41. Tee, Y. K. et al. Comparing different analysis methods for quantifying the MRI amide proton transfer (APT) effect in hyperacute stroke patients. NMR Biomed. 27, 1019-1029 (2014).

42. Walker-Samuel, S. et al. In vivo imaging of glucose uptake and metabolism in tumors. Nat. Med. 19, 1067-1072 (2013).

43. Tardif, C. L. et al. Advanced MRI techniques to improve our understanding of experienceinduced neuroplasticity. Neuroimage 131, 55-72 (2016).

44. Mugler, J. P. \& Brookeman, J. R. Three-dimensional magnetization-prepared rapid gradient-echo imaging (3D MP RAGE). Magn. Reson. Med. 15, 152-157 (1990).

45. Deoni, S. C. L., Peters, T. M. \& Rutt, B. K. High-resolution T1 and T2 mapping of the brain in a clinically acceptable time with DESPOT1 and DESPOT2. Magn. Reson. Med. 53, 237241 (2005).

46. Fram, E. K. et al. Rapid calculation of $\mathrm{T} 1$ using variable flip angle gradient refocused imaging. Magn. Reson. Imaging 5, 201-208 (1987).

47. Preibisch, C. \& Deichmann, R. T1 mapping using spoiled FLASH-EPI hybrid sequences and varying flip angles. Magn. Reson. Med. 62, 240-246 (2009).

48. Stikov, N. et al. On the accuracy of T1 mapping: searching for common ground. Magn. Reson. Med. 73, 514-522 (2015).

49. Mechelli, A., Friston, K. J., Frackowiak, R. S. \& Price, C. J. Structural covariance in the human cortex. J. Neurosci. 25, 8303-8310 (2005).

50. Lerch, J. P. et al. Mapping anatomical correlations across cerebral cortex (MACACC) using cortical thickness from MRI. Neuroimage 31, 993-1003 (2006).

51. Alexander-Bloch, A., Giedd, J. N. \& Bullmore, E. Imaging structural co-variance between human brain regions. Nat. Rev. Neurosci. 14, 322-336 (2013).

52. Evans, A. C. Networks of anatomical covariance. Neuroimage 80, 489-504 (2013).

53. Reid, A. T. et al. A seed-based cross-modal comparison of brain connectivity measures. 
Brain Struct. Funct. (2016). doi:10.1007/s00429-016-1264-3

54. Maguire, E. A. et al. Navigation-related structural change in the hippocampi of taxi drivers. Proc. Natl. Acad. Sci. U. S. A. 97, 4398-4403 (2000).

55. Draganski, B. et al. Neuroplasticity: changes in grey matter induced by training. Nature 427, 311-312 (2004).

56. Driemeyer, J., Boyke, J., Gaser, C., Büchel, C. \& May, A. Changes in Gray Matter Induced by Learning-Revisited. PLoS One 3, e2669 (2008).

57. Scholz, J., Klein, M. C., Behrens, T. E. J. \& Johansen-Berg, H. Training induces changes in white-matter architecture. Nat. Neurosci. 12, 1370-1371 (2009).

58. Sagi, Y. et al. Learning in the fast lane: new insights into neuroplasticity. Neuron 73, 11951203 (2012).

59. Taubert, M. et al. Dynamic properties of human brain structure: learning-related changes in cortical areas and associated fiber connections. J. Neurosci. 30, 11670-11677 (2010).

60. Hyde, K. L. et al. Musical training shapes structural brain development. J. Neurosci. 29, 3019-3025 (2009).

61. Bengtsson, S. L. et al. Extensive piano practicing has regionally specific effects on white matter development. Nat. Neurosci. 8, 1148-1150 (2005).

62. Thomas, C. \& Baker, C. I. Teaching an adult brain new tricks: a critical review of evidence for training-dependent structural plasticity in humans. Neuroimage 73, 225-236 (2013).

63. Thomas, A. G. et al. Functional but not structural changes associated with learning: an exploration of longitudinal voxel-based morphometry (VBM). Neuroimage 48, 117-125 (2009).

64. Lerch, J. P. et al. Maze training in mice induces MRI-detectable brain shape changes specific to the type of learning. Neuroimage 54, 2086-2095 (2011).

65. Blumenfeld-Katzir, T., Pasternak, O., Dagan, M. \& Assaf, Y. Diffusion MRI of structural brain plasticity induced by a learning and memory task. PLoS One 6, e20678 (2011). 
66. Scholz, J., Allemang-Grand, R., Dazai, J. \& Lerch, J. P. Environmental enrichment is associated with rapid volumetric brain changes in adult mice. Neuroimage 109, 190-198 (2015).

67. Sampaio-Baptista, C. \& Khrapitchev, A. A. Motor skill learning induces changes in white matter microstructure and myelination. The Journal of (2013).

68. Golub, Y., Kaltwasser, S. F., Mauch, C. P. \& Herrmann, L. Reduced hippocampus volume in the mouse model of Posttraumatic Stress Disorder. Journal of psychiatric (2011).

69. Keifer, O. P., Jr, Hurt, R. C., Gutman, D. A. \& Keilholz, S. D. Voxel-based morphometry predicts shifts in dendritic spine density and morphology with auditory fear conditioning. Nature (2015).

70. Biedermann, S. et al. In vivo voxel based morphometry: detection of increased hippocampal volume and decreased glutamate levels in exercising mice. Neuroimage 61, 1206-1212 (2012).

71. Fuss, J. et al. Exercise boosts hippocampal volume by preventing early age-related gray matter loss. Hippocampus 24, 131-134 (2014).

72. Jespersen, S. N. et al. Neurite density from magnetic resonance diffusion measurements at ultrahigh field: comparison with light microscopy and electron microscopy. Neuroimage 49, 205-216 (2010).

73. Sepehrband, F. et al. Brain tissue compartment density estimated using diffusion-weighted MRI yields tissue parameters consistent with histology. Hum. Brain Mapp. 36, 3687-3702 (2015).

74. Stikov, N. et al. In vivo histology of the myelin g-ratio with magnetic resonance imaging. Neuroimage 118, 397-405 (2015).

75. Jones, D. K., Knösche, T. R. \& Turner, R. White matter integrity, fiber count, and other fallacies: the do's and don'ts of diffusion MRI. Neuroimage 73, 239-254 (2013).

76. Streitbürger, D.-P. et al. Investigating structural brain changes of dehydration using voxel- 
based morphometry. PLoS One 7, e44195 (2012).

77. Trefler, A. et al. Impact of time-of-day on brain morphometric measures derived from T1weighted magnetic resonance imaging. Neuroimage 133, 41-52 (2016).

78. Satterthwaite, T. D. et al. Impact of in-scanner head motion on multiple measures of functional connectivity: relevance for studies of neurodevelopment in youth. Neuroimage 60, 623-632 (2012).

79. Reuter, M. et al. Head motion during MRI acquisition reduces gray matter volume and thickness estimates. Neuroimage 107, 107-115 (2015).

80. Alexander-Bloch, A. et al. Subtle in-scanner motion biases automated measurement of brain anatomy from in vivo MRI. Hum. Brain Mapp. 37, 2385-2397 (2016).

81. Yendiki, A., Koldewyn, K., Kakunoori, S., Kanwisher, N. \& Fischl, B. Spurious group differences due to head motion in a diffusion MRI study. Neuroimage 88, 79-90 (2014).

82. Pardoe, H. R., Kucharsky Hiess, R. \& Kuzniecky, R. Motion and morphometry in clinical and nonclinical populations. Neuroimage 135, 177-185 (2016).

83. Andersson, J. L. R. \& Sotiropoulos, S. N. An integrated approach to correction for offresonance effects and subject movement in diffusion MR imaging. Neuroimage 125, 10631078 (2016).

84. Chang, L.-C., Walker, L. \& Pierpaoli, C. Informed RESTORE: A method for robust estimation of diffusion tensor from low redundancy datasets in the presence of physiological noise artifacts. Magn. Reson. Med. 68, 1654-1663 (2012).

85. Andersson, J. L. R., Graham, M. S., Zsoldos, E. \& Sotiropoulos, S. N. Incorporating outlier detection and replacement into a non-parametric framework for movement and distortion correction of diffusion MR images. Neuroimage 141, 556-572 (2016).

86. Thesen, S., Heid, O., Mueller, E. \& Schad, L. R. Prospective acquisition correction for head motion with image-based tracking for real-time fMRI. Magn. Reson. Med. 44, 457-465 (2000). 
87. van der Kouwe, A., Fetics, B., Polenur, D., Roth, A. \& Nevo, E. Real-time prospective rigidbody motion correction with the EndoScout gradient-based tracking system. in Proc. 17th Scientific Meeting ISMRM 4623 (2009).

88. Ooi, M. B. et al. Combined prospective and retrospective correction to reduce motioninduced image misalignment and geometric distortions in EPI. Magn. Reson. Med. 69, 803811 (2013).

89. van Niekerk, A. M. J. et al. O51. A vector based approach for fast real time orientation measurement in magnetic resonance imaging (MRI). Phys. Med. 32, Supplement 2, 158 (2016/9).

90. Olesen, O. V., Paulsen, R. R., Højgaard, L., Roed, B. \& Larsen, R. Motion tracking for medical imaging: a nonvisible structured light tracking approach. IEEE Trans. Med. Imaging 31, 79-87 (2012).

91. Van der Kouwe, A. J. W., Benner, T. \& Dale, A. M. Real-time rigid body motion correction and shimming using cloverleaf navigators. Magn. Reson. Med. 56, 1019-1032 (2006).

92. Gallichan, D., Marques, J. P. \& Gruetter, R. Retrospective correction of involuntary microscopic head movement using highly accelerated fat image navigators (3D FatNavs) at 7T. Magn. Reson. Med. 75, 1030-1039 (2016).

93. Tisdall, M. D. et al. Prospective motion correction with volumetric navigators (vNavs) reduces the bias and variance in brain morphometry induced by subject motion. Neuroimage 127, 11-22 (2016).

94. Glasser, M. F. et al. The minimal preprocessing pipelines for the Human Connectome Project. Neuroimage 80, 105-124 (2013).

95. Uğurbil, K. et al. Pushing spatial and temporal resolution for functional and diffusion MRI in the Human Connectome Project. Neuroimage 80, 80-104 (2013).

96. Deoni, S. C. L. Correction of main and transmit magnetic field (B0 and B1) inhomogeneity effects in multicomponent-driven equilibrium single-pulse observation of T1 and T2. Magn. 
Reson. Med. 65, 1021-1035 (2011).

97. Umesh Rudrapatna, S., Juchem, C., Nixon, T. W. \& de Graaf, R. A. Dynamic multi-coil tailored excitation for transmit B1 correction at 7 Tesla. Magn. Reson. Med. 76, 83-93 (2016).

98. Watanabe, H., Takaya, N. \& Mitsumori, F. Non-uniformity correction of human brain imaging at high field by RF field mapping of B1+ and B1-. J. Magn. Reson. 212, 426-430 (2011).

99. van der Kouwe, A. J. W., Benner, T., Salat, D. H. \& Fischl, B. Brain morphometry with multiecho MPRAGE. Neuroimage 40, 559-569 (2008).

100.Pruessner, J. C. et al. Volumetry of temporopolar, perirhinal, entorhinal and parahippocampal cortex from high-resolution MR images: considering the variability of the collateral sulcus. Cereb. Cortex 12, 1342-1353 (2002).

101.Bookstein, F. L. 'Voxel-Based Morphometry' Should Not Be Used with Imperfectly Registered Images. Neuroimage 14, 1454-1462 (2001).

102.Ashburner, J. \& Friston, K. J. Why voxel-based morphometry should be used. Neuroimage $14,1238-1243$ (2001).

103. Fischl, B. et al. Cortical folding patterns and predicting cytoarchitecture. Cereb. Cortex 18, 1973-1980 (2008).

104. Mangin, J.-F. et al. A framework to study the cortical folding patterns. Neuroimage 23 Suppl 1, S129-38 (2004).

105.Eichner, C. et al. Slice accelerated diffusion-weighted imaging at ultra-high field strength. Magn. Reson. Med. 71, 1518-1525 (2014).

106. Vu, A. T. et al. High resolution whole brain diffusion imaging at $7 \mathrm{~T}$ for the Human Connectome Project. Neuroimage 122, 318-331 (2015).

107. Shmueli, K. et al. Magnetic susceptibility mapping of brain tissue in vivo using MRI phase data. Magn. Reson. Med. 62, 1510-1522 (2009). 
108.Schäfer, A. et al. Direct visualization of the subthalamic nucleus and its iron distribution using high-resolution susceptibility mapping. Hum. Brain Mapp. 33, 2831-2842 (2012).

109. Setsompop, K. et al. Blipped-controlled aliasing in parallel imaging for simultaneous multislice echo planar imaging with reduced g-factor penalty. Magn. Reson. Med. 67, 12101224 (2012).

110. Moeller, S. et al. Multiband multislice GE-EPI at 7 tesla, with 16 -fold acceleration using partial parallel imaging with application to high spatial and temporal whole-brain fMRI. Magn. Reson. Med. 63, 1144-1153 (2010).

111. Xu, J. et al. Evaluation of slice accelerations using multiband echo planar imaging at $3 \mathrm{~T}$. Neuroimage 83, 991-1001 (2013).

112. Hughes, E. J. et al. A dedicated neonatal brain imaging system. Magn. Reson. Med. (2016). doi:10.1002/mrm.26462

113. Setsompop, K. et al. Pushing the limits of in vivo diffusion MRI for the Human Connectome Project. Neuroimage 80, 220-233 (2013).

114. Sotiropoulos, S. N. et al. Advances in diffusion MRI acquisition and processing in the Human Connectome Project. Neuroimage 80, 125-143 (2013).

115.Fan, Q. et al. MGH-USC Human Connectome Project datasets with ultra-high b-value diffusion MRI. Neuroimage 124, 1108-1114 (2016).

116. McNab, J. A. et al. The Human Connectome Project and beyond: initial applications of 300 $\mathrm{mT} / \mathrm{m}$ gradients. Neuroimage 80, 234-245 (2013).

117. Ferizi, U. et al. White matter compartment models for in vivo diffusion MRI at $300 \mathrm{mT} / \mathrm{m}$. Neuroimage 118, 468-483 (2015).

118.Douaud, G. et al. In vivo evidence for the selective subcortical degeneration in Huntington's disease. Neuroimage 46, 958-966 (2009).

119. Panagiotaki, E. et al. Compartment models of the diffusion MR signal in brain white matter: a taxonomy and comparison. Neuroimage 59, 2241-2254 (2012). 
120. Jelescu, I. O. et al. In vivo quantification of demyelination and recovery using compartmentspecific diffusion MRI metrics validated by electron microscopy. Neuroimage $132,104-114$ (2016).

121.Kodiweera, C., Alexander, A. L., Harezlak, J., McAllister, T. W. \& Wu, Y.-C. Age effects and sex differences in human brain white matter of young to middle-aged adults: A DTI, NODDI, and q-space study. Neuroimage 128, 180-192 (2016).

122. Colgan, N. et al. Application of neurite orientation dispersion and density imaging (NODDI) to a tau pathology model of Alzheimer's disease. Neuroimage 125, 739-744 (2016).

123. Callaghan, P. T., Coy, A., MacGowan, D., Packer, K. J. \& Zelaya, F. O. Diffraction-like effects in NMR diffusion studies of fluids in porous solids. Nature 351, 467-469 (1991).

124.Shemesh, N., Ozarslan, E., Komlosh, M. E., Basser, P. J. \& Cohen, Y. From single-pulsed field gradient to double-pulsed field gradient MR: gleaning new microstructural information and developing new forms of contrast in MRI. NMR Biomed. 23, 757-780 (2010).

125.Kaden, E., Kruggel, F. \& Alexander, D. C. Quantitative mapping of the per-axon diffusion coefficients in brain white matter. Magn. Reson. Med. 75, 1752-1763 (2016).

126. Ozarslan, E. Compartment shape anisotropy (CSA) revealed by double pulsed field gradient MR. J. Magn. Reson. 199, 56-67 (2009).

127.Drobnjak, I., Zhang, H., lanuş, A., Kaden, E. \& Alexander, D. C. PGSE, OGSE, and sensitivity to axon diameter in diffusion MRI: Insight from a simulation study. Magn. Reson. Med. 75, 688-700 (2016).

128. Nilsson, M., van Westen, D., Ståhlberg, F., Sundgren, P. C. \& Lätt, J. The role of tissue microstructure and water exchange in biophysical modelling of diffusion in white matter. MAGMA 26, 345-370 (2013).

129. Westin, C.-F. et al. Q-space trajectory imaging for multidimensional diffusion MRI of the human brain. Neuroimage 135, 345-362 (2016).

130.Reisert, M., Kellner, E., Dhital, B., Hennig, J. \& Kiselev, V. G. Disentangling micro from 
mesostructure by diffusion MRI: A Bayesian approach. Neuroimage (2016).

doi:10.1016/j.neuroimage.2016.09.058

131.Lampinen, B. et al. Optimal experimental design for filter exchange imaging: Apparent exchange rate measurements in the healthy brain and in intracranial tumors. Magn. Reson. Med. (2016). doi:10.1002/mrm.26195

132.Devlin, J. T. \& Poldrack, R. A. In praise of tedious anatomy. Neuroimage 37, 1033-41; discussion 1050-8 (2007).

133. Margulies, D. S. et al. Situating the default-mode network along a principal gradient of macroscale cortical organization. Proc. Natl. Acad. Sci. U. S. A. (2016). doi:10.1073/pnas.1608282113

134.Collin, G., Sporns, O., Mandl, R. C. W. \& van den Heuvel, M. P. Structural and functional aspects relating to cost and benefit of rich club organization in the human cerebral cortex. Cereb. Cortex 24, 2258-2267 (2014).

135. Assaf, Y. \& Cohen, Y. Non-mono-exponential attenuation of water and N-acetyl aspartate signals due to diffusion in brain tissue. J. Magn. Reson. 131, 69-85 (1998).

136. Clark, C. A. \& Le Bihan, D. Water diffusion compartmentation and anisotropy at high b values in the human brain. Magn. Reson. Med. 44, 852-859 (2000).

137.Stanisz, G. J., Szafer, A., Wright, G. A. \& Henkelman, R. M. An analytical model of restricted diffusion in bovine optic nerve. Magn. Reson. Med. 37, 103-111 (1997).

138. Behrens, T. et al. Characterization and propagation of uncertainty in diffusion-weighted MR imaging. Magn. Reson. Med. 50, 1077-1088 (2003).

139. Assaf, Y. \& Basser, P. J. Composite hindered and restricted model of diffusion (CHARMED) MR imaging of the human brain. Neuroimage 27, 48-58 (2005).

140.Assaf, Y., Blumenfeld-Katzir, T., Yovel, Y. \& Basser, P. J. AxCaliber: a method for measuring axon diameter distribution from diffusion MRI. Magn. Reson. Med. 59, 13471354 (2008). 
141.Barazany, D., Basser, P. J. \& Assaf, Y. In vivo measurement of axon diameter distribution in the corpus callosum of rat brain. Brain 132, 1210-1220 (2009).

142. Alexander, D. C. et al. Orientationally invariant indices of axon diameter and density from diffusion MRI. Neuroimage 52, 1374-1389 (2010).

143. Tournier, J.-D., Mori, S. \& Leemans, A. Diffusion tensor imaging and beyond. Magn. Reson. Med. 65, 1532-1556 (2011).

144.Dell'Acqua, F., Simmons, A., Williams, S. C. R. \& Catani, M. Can spherical deconvolution provide more information than fiber orientations? Hindrance modulated orientational anisotropy, a true-tract specific index to characterize white matter diffusion. Hum. Brain Mapp. 34, 2464-2483 (2013).

145.Kaden, E., Knösche, T. R. \& Anwander, A. Parametric spherical deconvolution: inferring anatomical connectivity using diffusion MR imaging. Neuroimage 37, 474-488 (2007).

146. Sotiropoulos, S. N., Behrens, T. E. J. \& Jbabdi, S. Ball and rackets: Inferring fiber fanning from diffusion-weighted MRI. Neuroimage 60, 1412-1425 (2012).

147.Zhang, H., Schneider, T., Wheeler-Kingshott, C. A. \& Alexander, D. C. NODDI: practical in vivo neurite orientation dispersion and density imaging of the human brain. Neuroimage 61, 1000-1016 (2012).

148. Paus, T. Population Neuroscience. (Springer-Verlag, 2013).

149. Falk, E. B. et al. What is a representative brain? Neuroscience meets population science. Proceedings of the National Academy of Sciences 110, 17615-17622 (2013).

150.Paus, T. in Neuroepidemiology (eds. Rosano, C. I. M. \& Ganguli, M.) (Elsevier, 2016).

151. Thompson, P. M. et al. The ENIGMA Consortium: large-scale collaborative analyses of neuroimaging and genetic data. Brain Imaging Behav. 8, 153-182 (2014).

152.Psaty, B. M. et al. Cohorts for Heart and Aging Research in Genomic Epidemiology (CHARGE) Consortium: Design of prospective meta-analyses of genome-wide association studies from 5 cohorts. Circ. Cardiovasc. Genet. 2, 73-80 (2009). 
153. Odgers, C. L., Avshalom, C., Bates, C. J., Sampson, R. J. \& Moffitt, T. E. Systematic social observation of children's neighborhoods using Google Street View: a reliable and costeffective method. J. Child Psychol. Psychiatry 53, 1009-1017 (2012).

154. Mazziotta, J. et al. A probabilistic atlas and reference system for the human brain: International Consortium for Brain Mapping (ICBM). Philos. Trans. R. Soc. Lond. B Biol. Sci. 356, 1293-1322 (2001).

155.French, L. \& Paus, T. A FreeSurfer view of the cortical transcriptome generated from the Allen Human Brain Atlas. Front. Neurosci. 9, 323 (2015).

156. French, L. et al. Early Cannabis Use, Polygenic Risk Score for Schizophrenia and Brain Maturation in Adolescence. JAMA Psychiatry 72, 1002-1011 (2015).

157.Giedd, J. N. et al. Brain development during childhood and adolescence: a longitudinal MRI study. Nat. Neurosci. 2, 861-863 (1999).

158. Mills, K. L. et al. Structural brain development between childhood and adulthood: Convergence across four longitudinal samples. Neuroimage 141, 273-281 (2016).

159. Raznahan, A. et al. Longitudinal four-dimensional mapping of subcortical anatomy in human development. Proc. Natl. Acad. Sci. U. S. A. 111, 1592-1597 (2014).

160.Schmitt, J. E. et al. The dynamic role of genetics on cortical patterning during childhood and adolescence. Proc. Natl. Acad. Sci. U. S. A. 111, 6774-6779 (2014).

161. Shaw, P. et al. Intellectual ability and cortical development in children and adolescents. Nature 440, 676-679 (2006).

162. Shaw, P. et al. Attention-deficit/hyperactivity disorder is characterized by a delay in cortical maturation. Proc. Natl. Acad. Sci. U. S. A. 104, 19649-19654 (2007).

163. Raznahan, A. et al. Patterns of coordinated anatomical change in human cortical development: a longitudinal neuroimaging study of maturational coupling. Neuron 72, 873884 (2011).

164. Vandekar, S. N. et al. Topologically dissociable patterns of development of the human 
cerebral cortex. J. Neurosci. 35, 599-609 (2015).

165. Toro, R. et al. Brain volumes and Val66Met polymorphism of the BDNF gene: local or global effects? Brain Struct. Funct. 213, 501-509 (2009).

166. Reardon, P. K. et al. An Allometric Analysis of Sex and Sex Chromosome Dosage Effects on Subcortical Anatomy in Humans. J. Neurosci. 36, 2438-2448 (2016).

167.Lee, N. R. et al. Anatomical coupling among distributed cortical regions in youth varies as a function of individual differences in vocabulary abilities. Hum. Brain Mapp. 35, 1885-1895 (2014).

168. Alexander-Bloch, A. F. et al. Abnormal cortical growth in schizophrenia targets normative modules of synchronized development. Biol. Psychiatry 76, 438-446 (2014).

169. Honey, C. J. et al. Predicting human resting-state functional connectivity from structural connectivity. Proc. Natl. Acad. Sci. U. S. A. 106, 2035-2040 (2009).

170.Alexander-Bloch, A., Raznahan, A., Bullmore, E. \& Giedd, J. The convergence of maturational change and structural covariance in human cortical networks. J. Neurosci. 33, 2889-2899 (2013).

171.Seeley, W. W., Crawford, R. K., Zhou, J., Miller, B. L. \& Greicius, M. D. Neurodegenerative diseases target large-scale human brain networks. Neuron 62, 42-52 (2009).

172. Studholme, C. Mapping fetal brain development in utero using magnetic resonance imaging: the Big Bang of brain mapping. Annu. Rev. Biomed. Eng. 13, 345-368 (2011).

173. Kim, H. et al. NEOCIVET: Towards accurate morphometry of neonatal gyrification and clinical applications in preterm newborns. Neuroimage 138, 28-42 (2016).

174.Dubois, J. et al. Primary cortical folding in the human newborn: an early marker of later functional development. Brain 131, 2028-2041 (2008).

175.Anderson, P. J., Cheong, J. L. Y. \& Thompson, D. K. The predictive validity of neonatal MRI for neurodevelopmental outcome in very preterm children. Semin. Perinatol. 39, 147-158 (2015). 